\title{
A comparison of stock market mechanisms
}

\section{Giovanni Cespa*}

I analyze a multi-asset market under two trading mechanisms. In the first (the unrestricted system), traders' demand for each asset depends on all equilibrium prices and prices reflect the information contained in all order flows; in the second (the restricted system), traders' demand depends on the traded asset price and prices reflect single order flow information. I show that informed traders' use of multi-dimensional private information depends on the number of prices they observe, and on the price formation process. I then give conditions rendering the restricted system more efficient than the unrestricted system.

Keywords: Market Microstructure, Trading Mechanisms, Asset Pricing, Information and Market Efficiency.

JEL Classification: G100, G120, G140.

*Universitat Pompeu Fabra and CREA - Address: Departament d'Economia i Empresa, Ramon Trias Fargas, 25-27, E-08005 Barcelona. E-mail: giovanni.cespa@upf.edu

I thank Alberto Bennardo, Bruno Biais, Sandro Brusco, Jordi Caballé, Giacinta Cestone, Joseph Harrington (the Editor), Martin Hellwig, Marco Pagano, Frederic Palomino, Jozséf Sáckovics, Xavier Vives, Lucy White, Michael Wolf, and two anonymous referees for helpful comments and suggestions. The paper also benefitted from the comments of seminar audiences at Università di Salerno (CSEF), Universitat Pompeu Fabra, the 2001 CEPR European Summer Symposium in Financial Markets (Gerzensee), and the 2001 EFA Meeting (Barcelona). Support from the Barcelona Economics Program of CREA, and the EC through the TMR grants n. ERBFMBI-CT97-2528 and n. ERBFMRX-CT96-0054 is gratefully acknowledged. Part of this paper was written while I was visiting the Université des Sciences Sociales de Toulouse (GREMAQ), and the Ente per gli Studi Monetari e Finanziari "Luigi Einaudi" in Roma. The usual disclaimer applies. 


\section{Introduction}

The literature on stock market design has recently devoted attention to mechanisms allowing traders to exchange portfolios of assets. The idea behind these contributions is that the impossibility of operating in more than one market at the same time, a feature that characterizes virtually all of the existing stock markets, may either affect traders' capability to rebalance their portfolios (Bossaerts, Fine, and Ledyard, 2002) or seriously hamper their ability to exploit trade relevant information, and trigger program trades that cause price oscillations (Amihud and Mendelson 1991a; Amihud and Mendelson 1991b). A mechanism allowing the trade of asset portfolios would thus mitigate price volatility and permit better portfolio re-balancing.

From the perspective of market design it is then important to understand how to concretely implement such a trading system. Consider, for instance, a trader submitting an order to buy a given vector of assets. She may want to condition her demand not only on the price of the asset she is trading, but also to take advantage of cross-conditioning possibilities. In particular, she may want to condition her decision to buy say a hundred shares of company $A$ both on the price of company $A$ and on that of company $B$, to the extent that information flows about the two companies are somewhat related. This type of cross-conditioning has been advocated by many authors on grounds of improved efficiency and reduced volatility (Beja and Hakansson, 1979; Amihud and Mendelson, 1991b; Economides and Schwartz, 1995). Surprisingly, little theoretical analysis has assessed the desirability of its introduction.

Aside from theoretical considerations, this analysis is prompted by the deep changes in trading procedures spurred by recent advances in information technology. ITG, the technology company running the POSIT network, has recently started allowing its clients the submission of multi-price 
contingent orders. 1 Optimark, a trading system directed to institutional traders, allowed the specification of different parameters upon which to condition trade execution. ${ }^{2}$ Archipelago, an open limit order book system, allows participants to submit non standard types of orders. 3

Motivated by these considerations, I analyze the properties of two call-auction trading mechanisms in which a vector of (two) risky assets is traded among a continuum of risk-averse informed speculators and liquidity traders, with the intermediation of a competitive, risk-neutral marketmaking sector. Informed traders receive a vector of private (noisy) signals about the vector of liquidation values and, in the unrestricted mechanism, submit multi-price contingent orders; in the restricted mechanism they submit standard (single-price contingent) limit orders. Market makers in the unrestricted mechanism compete for each asset order flow, whereas in the restricted mechanism their competition is restricted to the order flow of the asset they are assigned to. In both cases, equilibrium prices are set equal to the expected value of the risky asset conditional on all public information. Liquidity traders are modeled as having a vector of random demands. All random variables are normally distributed and informed traders display a constant absolute risk-aversion utility function. Equilibrium behavior is analyzed and implications for price informativeness and traders' welfare are addressed.

Contrary to common intuition, this analysis challenges the view that a multi-price contingent

\footnotetext{
${ }^{1}$ The electronic equity-matching system ITG started operating 14 years ago. Its trading platform QuantEX permits an order submission strategy ("Pairs") that automatically executes orders "when the spread differential between two stocks reaches a specified level." QuantEX, Electronic Trading Made Intelligent, available at http: / www. itginc.com. I thank Ekkehart Boehmer for pointing out this evidence to me.

${ }^{2}$ Besides submitting traditional limit and market orders, traders could condition their demand on a number of contingencies. For instance, a trader could specify her willingness to pay more for a larger order size in a confidential way, so that the actual transaction price would not be affected. See Clemons and Weber (1998).

${ }^{3}$ For instance, traders can post "discretionary orders," where they specify both a limit price and the price difference they are willing to accept to get the order executed (for instance, a trader may want to buy 1000 shares at $\$ 10$ but may be willing to pay $\$ 10_{1 / 4}$ at most. The order is posted at $\$ 10$ and if a sell at $\$ 10^{1 / 4}$ enters the book, it is executed). Also, they can post tracking orders that are automatically adjusted to the National Best Bid and Offer (NBBO) changes. See Wall Street Letter, December 4, 2000. For a survey of recent trading platforms' innovations see the Economist, May, 18th 2000.
} 
system should always render the market more efficient. Amihud and Mendelson (1991b, p. 127) argue that "a mechanism which enables simultaneous conditioning of orders for different assets (...) would increase the information available to traders, improve value discovery and reduce volatility." This assertion points at the positive effect that observing multiple sources of correlated information has. By contrast, my paper unveils the dark side of a multi-price contingent system, by analyzing its feedback effect on traders' speculative aggressiveness.

A fundamental insight of a multi-asset market is that a trader's use of multi-dimensional private information depends on the type of order she submits, and on the amount of information market makers observe (which, as a consequence, is reflected by equilibrium prices). In the unrestricted system, market makers set prices conditionally on the vector of all order flows; thus, all the crossorder flow information about fundamentals is already reflected into prices, and traders do not find such information useful to improve their position vis-à-vis market makers. However, market makers cannot observe the signals informed traders receive. Therefore, insofar as private signal error terms are correlated, traders use multi-signal conditioning to disentangle error terms from fundamentals in their private signals. Conversely, in the restricted system, market makers set prices conditionally on the observation of the asset order flow they are assigned to. Hence, equilibrium prices only partially reflect cross-order flow information about fundamentals. This, in turn, renders multiple private signals useful to informed traders beyond the correlated information about error terms they contain. The upshot is that whenever private signal error terms are independent, traders speculate more aggressively on their private information in the restricted system than in the unrestricted system. This implies that, when the information structure is homoscedastic, if order flows are correlated only through fundamentals, the restricted system delivers more informative prices than the unrestricted system. 
The central idea behind the efficiency result is that differently from a single-asset framework, in a multi-asset setup price informativeness depends on two factors: the correlation between each order flow and the asset payoff and the correlation across order flows. Indeed, the more correlated with the fundamentals the order flows are, the more fundamental information can be extracted by observing them; also, the more correlated order flows are among themselves, the easier it is to disentangle noise from information within each order flow. Due to collinearity effects, the interplay between these two factors may impair price informativeness in both trading systems. However, this problem is neutralized in the restricted system as the stronger aggressiveness traders exhibit magnifies the effect of the second factor, boosting price informativeness. This, on the other hand, comes at the cost of making the price impact of trades harsher and, thus, noise traders' expected losses higher.

There is by now a vast literature studying the effects of different trading mechanisms on agents' behavior and market patterns. However, most of it has concentrated on the analysis of single (risky) asset markets. Madhavan (1992) compares the properties of quote driven systems with those of order driven systems. Biais (1993) contrasts centralized and fragmented markets. Pagano and Röell (1996) assess the effects of market transparency on uninformed traders' losses. Grossman (1992), in a paper which is closely related to the present one, justifies the coexistence of upstairs and downstairs markets. He argues that, contrary to what economic theory usually assumes, technical limitations prevent investors from expressing their demands as a function of a price vector, and from continuously updating them as new information arrives. This precludes investors' preferences from being accurately represented on organized markets, and gives upstairs dealers, acting as repositories of information about unexpressed demands, a transaction cost advantage vis-à-vis downstairs dealers. In view of this paper's results, and insofar as a major function stock markets perform is to 
signal firms' true assets' payoffs, overcoming technological limitations may not always be a good idea, as it can impair price efficiency. 4

Little is known about the properties of markets where traders' private information is multidimensional. A notable exception is the paper by Manzano (1997) where the author compares multi-price and single-price contingent systems in a model with strategic traders. Also related are the analyses of Wohl and Kandel (1997) and Brown and Holden (2002). These papers study a trading mechanism where traders condition their demand for a given asset on a market index. Hence, their focus is rather on the advantages of avoiding mispricing risk. ${ }^{5}$ However, none of the above papers assesses the effect that observing multiple sources of endogenous public information (i.e. equilibrium prices) has on the use that traders make of multidimensional private information, and on price efficiency.

The paper is organized as follows. In the next section, I compare a one-asset market where traders submit limit orders to one where they submit market orders. This provides a useful benchmark on which to build the comparison of market mechanisms in the multi-asset setup. In the third section, I characterize the unique linear equilibria of the two mechanisms. In the fourth section, I compare their properties. In the fifth section I consider two extensions of the multi-asset model, generalizing the structure of private information, and introducing an intermediate mechanism where traders' orders are restricted while market-makers are able to observe multiple order flows. The sixth section concludes the paper. Most of the proofs are relegated to the final appendices.

\footnotetext{
${ }^{4}$ See Fishman and Hagerty (1992) for a discussion of the importance of stock price efficiency for production decisions within and outside the firm.

${ }^{5}$ Mispricing risk is the risk that a limit order is executed at a mispriced limit price (as is the case, e.g. when some relevant information is revealed to the market and the limit price is not updated to take it into account).
} 


\section{The benchmark: limit orders vs. market orders}

In this section I compare the properties of two markets where all the informed traders either submit limit orders or submit market orders. As will become clear later, insofar as traders in the restricted system fail to condition their demand on all the sources of information related to the fundamentals (as they do when submitting a market order), this analysis provides a useful benchmark on which to build the comparison of market mechanisms in the multi-asset setup. 6

In both markets a single risky asset with liquidation value $v \sim N\left(\bar{v}, \tau_{v}^{-1}\right)$ and a riskless asset with unitary return, are traded among risk averse informed speculators and noise traders with the intermediation of a competitive, risk neutral market making sector. There is a continuum of informed traders in the interval $[0,1]$. Each informed trader $k$ receives a private signal $s_{k}=v+\epsilon_{k}$ about the unknown $v$, where $\epsilon_{k} \sim N\left(0, \tau_{\epsilon}^{-1}\right)$, and $\epsilon_{k}, \epsilon_{h}$ are independent for $k \neq h$. Assume that her preferences are represented by a CARA utility $U\left(\pi_{k}\right)=-\exp \left\{-\pi_{k} / \gamma\right\}$ where $\gamma>0$ denotes the coefficient of constant absolute risk tolerance and $\pi_{k}=x_{k}(v-p)$ indicates the profit of buying $x_{k}$ units of the asset at price $p$. Normalize the informed traders' initial wealth to zero and let noise traders submit a random demand $u \sim N\left(0, \tau_{u}^{-1}\right)$. Finally, assume that the random variables $v, u, \epsilon_{k}$ are independent $\forall k$ and that, given $v$, the average signal $\int_{0}^{1} s_{k} d k$ equals almost surely $v$ (i.e. errors cancel out in the aggregate: $\left.\int_{0}^{1} \epsilon_{k} d k=0\right)$.

\section{The "limit order" market}

Suppose that all informed traders submit limit orders. Therefore, every trader $k$ submits a schedule $X_{L k}\left(s_{k}, p\right)$ indicating her desired position in the risky asset contingent on her private signal and on

\footnotetext{
${ }^{6}$ Thus, this analysis does not provide a theory of order flow composition as all informed traders are assumed to make the same choice as to the type of order they submit in a market populated by risk neutral market makers and liquidity traders. For a theory of order flow composition see Foucault (1999).
} 
the price. I restrict my attention to linear equilibria where $X_{L k}\left(s_{k}, p\right)=a_{L} s_{k}+b_{L} p$. Competitive, risk neutral market makers set a semi-strong efficient equilibrium price conditional on the observation of the order flow $L(p)=\int_{0}^{1} X_{L k}\left(s_{k}, p\right) d k+u=a_{L} v+u+b_{L} p$. Let $z_{L}=a_{L} v+u$ denote the informational content of the order flow. Then, $p=E\left[v \mid z_{L}\right]$ and the following result applies

Proposition 1 (Vives (1995b)) In the limit order market there is a unique linear equilibrium. It is symmetric and given by $X_{L k}\left(s_{k}, p\right)=a_{L}\left(s_{k}-p\right)$ and $p=\lambda_{L} z_{L}+\left(1-\lambda_{L} a_{L}\right) \bar{v}$, where $a_{L}=\gamma \tau_{\epsilon}$, $\lambda_{L}=a_{L} \tau_{u} / \tau_{L}$ and $\tau_{L}=\left(\operatorname{Var}\left[v \mid z_{L}\right]\right)^{-1}=\tau_{v}+a_{L}^{2} \tau_{u}$.

Intuitively, informed speculators' trading aggressiveness in the limit order market $a_{L}$ increases in the precision of their private signal and in the risk tolerance coefficient. Market makers' reaction to the presence of informed speculators $\lambda_{L}=a_{L} \tau_{u} / \tau_{L}$ is captured by the OLS regression coefficient of the unknown payoff value on the order flow. As common in this literature $\lambda_{L}$ measures the reciprocal of market depth (see e.g. Kyle, 1985 and Vives 1995b, 1995a). The informativeness of the equilibrium price is measured by the reciprocal of the payoff conditional variance given the order flow: $\left(\operatorname{Var}\left[v \mid z_{L}\right]\right)^{-1}=\tau_{L}$. The higher $\tau_{L}$, the smaller the uncertainty on the true payoff value once the order-flow has been observed.

\section{The "market order" market}

Suppose instead all informed traders submit market orders. Thus, assume each trader $k$ submits a schedule $X_{M k}\left(s_{k}\right)$ indicating her desired position contingent on the private signal she receives, and restrict attention to linear equilibria where $X_{M k}\left(s_{k}\right)=a_{M} s_{k}+b_{M}$. Competitive, risk neutral market makers set a semi-strong efficient equilibrium price conditional on the observation of the 
order flow $L=\int_{0}^{1} X_{M k}\left(s_{k}\right) d k+u=a_{M} v+u+b_{M} \cdot{ }^{7}$ Let $z_{M}=a_{M} v+u$ denote the informational content of the order flow. Then, $p=E\left[v \mid z_{M}\right]$ and the following result applies

Proposition 2 (Vives (1995a)) In the market order market there is a unique linear equilibrium. It is symmetric and given by $X_{M k}\left(s_{k}\right)=a_{M}\left(s_{k}-\bar{v}\right)$ and $p=\lambda_{M} z_{M}+\left(1-\lambda_{M} a_{M}\right) \bar{v}$, where $a_{M}=\gamma\left(\tau_{\epsilon}^{-1}+\operatorname{Var}[p]\right)^{-1}$ is the unique positive root of the cubic equation $F\left(a_{M}\right)=\left(\left(a_{M} / \gamma \tau_{\epsilon}\right)-\right.$ 1) $\tau_{v}+\left(\lambda_{M} / \gamma\right) a_{M}^{2}=0$, with $\lambda_{M}=a_{M} \tau_{u} / \tau_{M}$ and $\tau_{M}=\left(\operatorname{Var}\left[v \mid z_{M}\right]\right)^{-1}=\tau_{v}+a_{M}^{2} \tau_{u}$.

Informed speculators' trading aggressiveness in the market order market $a_{M}$ is inversely related to the ex-ante volatility of the price $\operatorname{Var}[p]$. Indeed, while traders condition on private information, they do not anticipate the equilibrium price. Thus, the larger the equilibrium price variance, the higher the execution risk, i.e. the risk of having their order executed at a price different from the one prevailing when they submitted it, and the smaller $a_{M}$.

\section{Comparing limit orders with market orders}

Given the previous results, we can now compare traders' behavior, market performance and traders' welfare in the two markets. Indicate with $\operatorname{Var}\left[p ; a_{L}\right]$ and $\operatorname{Var}\left[p ; a_{M}\right]$ respectively the ex-ante price volatility in the limit order market and in the market order market.

\section{Proposition 3}

1. Informed traders in the market order market trade less aggressively than in the limit order market: $a_{M}<a_{L}$; as a result prices in the market order market are less informative and ex-ante less volatile than in the limit order market: $\tau_{M}<\tau_{L}$ and $\operatorname{Var}\left[p ; a_{M}\right]<\operatorname{Var}\left[p ; a_{L}\right]$.

\footnotetext{
${ }^{7}$ An equivalent interpretation of this market is one where prices are set through a market clearing process, the competitive market making sector submits limit orders while informed and noise traders submit market orders to a centralized auctioneer (see Vives, 1995a).
} 
2. The market order market is deeper than the limit order market if and only if $a_{M} / a_{L}<\tau_{M} / \tau_{L}$.

Proof. The first part follows immediately from the definitions of $a_{M}$ and $a_{L}$, since $a_{M} \equiv \gamma\left(\tau_{\epsilon}^{-1}+\right.$ $\left.\operatorname{Var}\left[p ; a_{M}\right]\right)^{-1}<\gamma \tau_{\epsilon} \equiv a_{L}$. Given this, $\tau_{M}<\tau_{L}$ and owing to price efficiency $\operatorname{Var}\left[p ; a_{M}\right]=$ $\tau_{v}^{-1}-\tau_{M}^{-1}<\tau_{v}^{-1}-\tau_{L}^{-1}=\operatorname{Var}\left[p ; a_{L}\right]$. Part 2 follows from the definition of $\lambda_{M}$ and $\lambda_{L}$. It is immediate to see that there are values of the parameters for which $\lambda_{M}<\lambda_{L}$ as rearranging this inequality leads to $\left(a_{M}-a_{L}\right)\left(\tau_{v}-a_{M} a_{L} \tau_{u}\right)<0$. As $a_{M}<a_{L}$, for this condition to hold it must be that $\tau_{v}>a_{M} a_{L} \tau_{u}$. Suppose this is never possible, i.e. $\tau_{v} / a_{L} \tau_{u} \leq a_{M}$, then as $a_{M}<a_{L}$, this implies $\tau_{v} / \tau_{u}<a_{L}^{2}$ which is clearly not always true.

The intuition for the above results is straightforward: risk averse informed speculators in the market order market suffer from execution risk. As a consequence, they scale back their aggressiveness compared with speculators in the limit order market. Therefore, they embed less information in the order flow, lowering the market order market price informativeness. 8 Indicating with $\rho_{v, z_{M}}$ the correlation coefficient between the informational content of the order flow and the asset payoff in the market order market it is immediate to show that

$$
\operatorname{Var}\left[p ; a_{M}\right]=\tau_{v}^{-1}-\tau_{M}^{-1}=\tau_{v}^{-1} \rho_{v, z_{M}}^{2}
$$

Hence, in a semi-strong efficient market, ex-ante price volatility reflects the arrival of information (analogously in the limit order market $\operatorname{Var}\left[p ; a_{L}\right]=\tau_{v}^{-1} \rho_{v, z_{L}}^{2}$ ). The stronger is the correlation between the informational content of the order flow and the fundamentals (i.e. the more informative is the price about the liquidation value), the higher is the ex-ante volatility of the price.

\footnotetext{
${ }^{8}$ This result thus contrasts with Rochet and Vila (1994), who in their analysis of Kyle (1985) show that price informativeness does not depend on the type of order the insider submits. The reason is that in their model strategic behavior leads the limit order insider to scale down her aggressiveness, equalizing the amount of information flowing to the market to that of the market order model. In the present context, no strategic effects arise while risk aversion translates execution risk into a trading aggressiveness reduction.
} 
Comparing depth across the two markets, two effects are at play: first, as $a_{M}<a_{L}$, market makers' adverse selection problem is less severe in the market order market; second, since $\tau_{M}<\tau_{L}$, market makers in the market order market are less able to disentangle noise from information. If the positive effect coming from the reduction in traders' aggressiveness is stronger than the negative effect due to the reduction in transparency, the market order market is deeper.

Indicate with $\operatorname{Var}\left[v-p \mid s_{k} ; a_{M}\right]\left(\operatorname{Var}\left[v-p \mid p, s_{k} ; a_{L}\right]\right)$ the variance of the returns conditional on private information in the market (limit) order market and with $\operatorname{Var}\left[v-p \mid p ; a_{M}\right]\left(\operatorname{Var}\left[v-p \mid p ; a_{L}\right]\right)$ the variance of the returns conditional on the order flow in the market (limit) order market.

\section{Proposition 4}

1. An informed trader $k$ prefers to trade in the limit order market rather than in the market order market if and only if $\left(\operatorname{Var}\left[v-p \mid p ; a_{L}\right]\right)^{-1 / 2} \operatorname{Var}\left[v-p \mid p, s_{k} ; a_{L}\right]^{1 / 2}<\left(\operatorname{Var}\left[v-p \mid p ; a_{M}\right]\right)^{-1 / 2}$ $\operatorname{Var}\left[v-p \mid s_{k} ; a_{M}\right]^{1 / 2}$

2. Noise traders' expected losses are larger in the limit order market if and only if $a_{M} / a_{L} \leq$ $\tau_{M} / \tau_{L}$

Proof. Standard normal calculations give $E\left[-\exp \left\{-\gamma^{-1} x_{M k}(v-p)\right\}\right]=E\left[E\left[-\exp \left\{-\gamma^{-1} x_{M k}\right.\right.\right.$ $\left.\left.(v-p)\} \mid s_{k}\right]\right]=E\left[-\exp \left\{-\gamma^{-1}\left(E\left[x_{M k}(v-p) \mid s_{k}\right]-(1 / 2 \gamma) \operatorname{Var}\left[x_{M k}(v-p) \mid s_{k}\right]\right)\right\}\right]=E[-\exp \{-(1 / 2)$ $\left.\left.\operatorname{Var}\left[v-p \mid s_{k}\right]^{-1} E\left[v-p \mid s_{k}\right]^{2}\right\}\right]$, and applying lemmaßB1, $E\left[-\exp \left\{-\gamma^{-1} x_{M k}(v-p)\right\}\right]=(\operatorname{Var}[v-$ $\left.\left.p \mid p ; a_{M}\right]\right)^{-1 / 2} \operatorname{Var}\left[v-p \mid s_{k} ; a_{M}\right]^{1 / 2}$. Similarly, $E\left[-\exp \left\{-\gamma^{-1} x_{L k}(v-p)\right\}\right]=-\left(\tau_{L} /\left(\tau_{L}+\tau_{\epsilon}\right)\right)^{1 / 2}$ $=\left(\operatorname{Var}\left[v-p \mid p ; a_{L}\right]\right)^{-1 / 2} \operatorname{Var}\left[v-p \mid p, s_{k} ; a_{L}\right]^{1 / 2}$. For part 2, $E[u(v-p)]=-\lambda_{M} \tau_{u}^{-1}$ in the market order market and $E[u(v-p)]=-\lambda_{L} \tau_{u}^{-1}$ in the limit order market. The result follows. Q.E.D.

Thus, the ex-ante expected utility that an informed trader earns submitting a market (limit) order, depends on the informational advantage she retains over the market makers. The smaller is 
the volatility of returns, given the trader information, vis-à-vis the volatility of returns, given the market maker information, the more precise is traders' estimate of asset returns compared to market makers'. For a given payoff volatility and noise traders' demand dispersion, the condition in the proposition is satisfied whenever the quality of private information is poor, and traders are very risk averse $\left(\tau_{v}=\tau_{u}=\tau_{\epsilon}=\gamma=.1\right)$. If this is the case, submitting a limit order (i.e. drawing inferences from the price) improves the precision of a trader's forecast without dissipating too much information to the benefit of market makers. As the quality of information improves and traders become more risk tolerant (e.g. $\tau_{v}=\tau_{u}=.1, \tau_{\epsilon}=4$, and $\gamma=1$ ), the reverse occurs, as the higher aggressiveness traders display submitting a limit order makes them loose most of their informational advantage to market makers. In this situation, a trader rather submits a market order.

As noise traders' expected losses are inversely proportional to market depth, whenever the market order market is deeper than the limit order market, noise traders experience lower expected losses in that market.

\section{Multi-asset vs. single-asset trading mechanisms}

In this section I extend the assumptions of the previous section to a two-asset setup. For the notation let us indicate with $\boldsymbol{\Pi}_{\boldsymbol{x}}$ the precision matrix of the two-dimensional random vector $\boldsymbol{x}$; with $\tau_{x_{i}}$ the precision of the random variable $x_{i}$ and with $\rho_{x}$ the correlation coefficient of the random vector $\left(x_{1}, x_{2}\right)$. Suppose that informed and noise traders exchange a vector of $t w o$ risky assets with random liquidation value $\boldsymbol{v}=\left(v_{1}, v_{2}\right) \sim N\left(\overline{\boldsymbol{v}}, \boldsymbol{\Pi}_{\boldsymbol{v}}^{-1}\right)$ and a riskless one with unitary return with the intermediation of a competitive, risk neutral market making sector. There is a continuum of informed traders in the interval $[0,1]$. Each informed trader $k$ receives a vector of private signals $\boldsymbol{s}_{k}=\boldsymbol{v}+\boldsymbol{\epsilon}_{k}$ about the unknown $\boldsymbol{v}$, where $\boldsymbol{\epsilon}_{k}=\left(\epsilon_{k 1}, \epsilon_{k 2}\right) \sim N\left(\mathbf{0}, \boldsymbol{\Pi}_{\boldsymbol{\epsilon}}^{-1}\right)$, and $\boldsymbol{\epsilon}_{k}$ and $\boldsymbol{\epsilon}_{h}$ are independent for 
$k \neq h$. Assume that her preferences are represented by a CARA utility $U\left(\pi_{k}\right)=-\exp \left\{-\pi_{k} / \gamma\right\}$ where $\gamma>0$ indicates the coefficient of constant absolute risk tolerance and $\pi_{k}=\boldsymbol{x}_{k}^{\prime}(\boldsymbol{v}-\boldsymbol{p})$ denotes the profit of buying $\left(x_{k 1}, x_{k 2}\right)$ units of each asset at price $\boldsymbol{p}$. Normalize the informed traders' initial wealth to zero and let noise traders submit a random demand $\boldsymbol{u}=\left(u_{1}, u_{2}\right) \sim N\left(\mathbf{0}, \boldsymbol{\Pi}_{\boldsymbol{u}}^{-1}\right)$. Assume that the random vectors $\boldsymbol{v}, \boldsymbol{u}, \boldsymbol{\epsilon}_{k}$ are independent $\forall k$ and that given $\boldsymbol{v}$, the vector of average signals $\int_{0}^{1} \boldsymbol{s}_{k} d k$ equals almost surely $\boldsymbol{v}$. Finally, let each of $\boldsymbol{\Pi}_{\boldsymbol{v}}^{-1}, \boldsymbol{\Pi}_{\boldsymbol{u}}^{-1}$, and $\boldsymbol{\Pi}_{\boldsymbol{\epsilon}}^{-1}$ be positive definite and suppose that the distributional assumptions are common knowledge among the agents in the economy.

With the above assumptions, I consider two market mechanisms:

1. the unrestricted mechanism where (a) speculators condition their demand for each asset $j$ on the vector of private signals $s_{k}$ and on the price of assets $j=1,2$, and (b) market makers set the price of asset $j$ conditionally on the observation of the order flow of both assets $j=1,2$;

2. the restricted mechanism where (a) speculators condition their demand for an asset $j$ on the vector of private signals $s_{k}$ and on the price of asset $j$ only and (b) market makers set the price of asset $j$ conditionally on the observation of the order flow $j$. In this case, interpreting market makers as uninformed speculators, the model captures the features of the opening auction in those markets where traders are allowed to condition their demand for an asset only on its own price.

\section{The unrestricted system}

The unrestricted system is a version of the multi-asset model of Admati (1985) with the addition of a risk-neutral, competitive, market-making sector as in Vives (1995b). 9

\footnotetext{
${ }^{9}$ For noisy rational expectations equilibrium models with a single risky asset see Hellwig (1980), Diamond and Verrecchia (1981) and Grossman and Stiglitz (1980).
} 
Suppose informed traders submit multi-price contingent orders. Thus, each trader $k$ submits a vector of demand schedules $\mathbf{X}_{k}\left(\boldsymbol{s}_{k}, \boldsymbol{p}\right)$, indicating the position desired in each asset $j$ at every price vector $\boldsymbol{p}$, contingent on the available private information. I restrict my attention to linear equilibria. In equilibrium, then, prices will be normally distributed.

Market makers observe the vector of aggregate order flows $\mathbf{L}(\boldsymbol{p})=\int_{0}^{1} \mathbf{X}_{k}\left(\boldsymbol{s}_{k}, \boldsymbol{p}\right) d k+\boldsymbol{u}$. Therefore, in pricing asset $j$ each market maker uses both the information contained in order flow $j$ and that contained in order flow $i \neq j$. Owing to the assumed ex-ante symmetric information structure, the vector of demand functions and the equilibria will be symmetric. Suppose then that $\mathbf{X}_{k}\left(\boldsymbol{s}_{k}, \boldsymbol{p}\right)=\mathbf{A} \boldsymbol{s}_{k}+\phi(\boldsymbol{p})$, where $\mathbf{A}$, and $\phi(\cdot)$ are, respectively, the matrix of trading intensities and a linear function of current prices. The aggregate order flow is then given by $\mathbf{L}(\boldsymbol{p})=\boldsymbol{z}+\phi(\boldsymbol{p})$, where $z=\mathbf{A} \boldsymbol{v}+\boldsymbol{u}$, denotes the vector of order flows' informational contents. Owing to competition for each order flow and risk neutrality, market makers set a semi-strong efficient price vector $\boldsymbol{p}=E[\boldsymbol{v} \mid \boldsymbol{z}]=\boldsymbol{\Pi}^{-1}\left(\boldsymbol{\Pi}_{\boldsymbol{v}} \overline{\boldsymbol{v}}+\mathbf{A}^{\prime} \boldsymbol{\Pi}_{\boldsymbol{u}} \boldsymbol{z}\right)$, where $\boldsymbol{\Pi}=\boldsymbol{\Pi}_{\boldsymbol{v}}+\mathbf{A}^{\prime} \boldsymbol{\Pi}_{\boldsymbol{u}} \mathbf{A}$, and the following result holds:

Proposition 5 In the unrestricted system there exists a unique equilibrium in linear strategies. It is symmetric and given by

$$
\mathbf{X}_{k}\left(s_{k}, \boldsymbol{p}\right)=\mathbf{A}\left(s_{k}-\boldsymbol{p}\right),
$$

and $\boldsymbol{p}=\boldsymbol{\Lambda} \boldsymbol{z}+(I-\boldsymbol{\Lambda} \mathbf{A}) \overline{\boldsymbol{v}}$, where $\mathbf{A}=\gamma \boldsymbol{\Pi}_{\boldsymbol{\epsilon}}$ and $\boldsymbol{\Lambda}=\boldsymbol{\Pi}^{-1} \mathbf{A}^{\prime} \boldsymbol{\Pi}_{\boldsymbol{u}}$.

Proof. See Appendix A.

Remark 1 The matrix $\Lambda$ maps order flows into prices. For the equilibrium to be well-defined, $\boldsymbol{\Lambda}$ must be invertible and, given the model's assumptions, this is always the case. Notice also that, 
owing to multicollinearity effects, the diagonal elements of this matrix can be negative (see Admati, 1985).

The next corollary characterizes how speculators use public and private information in equilibrium.

Corollary 1 In the unrestricted system, an informed speculator's demand for each asset $j=1,2$, depends on the whole private signal vector $s_{k}$ and on the whole price vector $p$ if and only if $\rho_{\epsilon} \neq 0$.

Proof. Follows from the fact that $\mathbf{A}=\gamma \boldsymbol{\Pi}_{\boldsymbol{\epsilon}}$.

Corollary 1 highlights a fundamental property of the unrestricted system: informed traders' multi-price (and signal) conditioning is optimal if and only if the private signals' conditional precision matrix is not diagonal. The intuition is as follows. As market makers observe both order flows, equilibrium prices reflect all cross-order flow information about the fundamentals. Hence, informed traders do not find such information useful to improve their position vis-à-vis market makers. However, market makers cannot observe the signals informed traders receive. Therefore, insofar as error terms are correlated, traders use multi-price (and multi-signal) conditioning to disentangle error terms from fundamentals within their private signals.

Remark 2 Writing in scalar form a trader's strategy one can see that the trading intensity in an asset $j$ is the composition of two effects: a direct one stemming from the informational advantage the speculator has over the rest of the market in asset $j$, and an indirect one coming from the informational advantage she has on the other asset, to the extent that the received signals are correlated. To see this, indicate with $\tau_{\epsilon_{j}}, j=1,2$ the (conditional) signal precision in asset $j$. Then, the strategy 
of a speculator in asset $j$ can be written as follows:

$$
X_{k j}\left(\boldsymbol{s}_{k}, \boldsymbol{p}\right)=\frac{\gamma \tau_{\epsilon_{j}}}{\left(1-\rho_{\epsilon}^{2}\right)}\left(s_{k j}-p_{j}\right)-\frac{\gamma \rho_{\epsilon} \sqrt{\tau_{\epsilon_{j}} \tau_{\epsilon_{i}}}}{\left(1-\rho_{\epsilon}^{2}\right)}\left(s_{k i}-p_{i}\right)
$$

Assume that $\rho_{\epsilon}>0$ and that speculator $k$ receives two signals $s_{k j}, s_{k i}$ such that $s_{k j}>p_{j}$ and $s_{k i}>p_{i}$. This can happen for two reasons: either both assets are worth more than what the market thinks (i.e. asset prices are biased downwards e.g. by noise traders' selling pressure); or both signals are biased upwards. A downward bias in equilibrium prices is good news since it gives the trader the possibility of taking advantage of the market's forecast error. Her demand in each asset is larger, the more precise are the signals she has received. However, the existence of positive correlation across signal-error terms strengthens the hypothesis of a contemporaneous, upward bias in the speculator's signals. Given this, the speculator reinforces her belief that the good news she received about both assets is due to the effect of error terms and reduces her demand in both asset $j$ and asset $i .10$

When no correlation across error terms exists $\left(\rho_{\epsilon}=0\right)$, speculators have no way to reduce the bias in their strategies by pooling together private signals and find it optimal to submit single-signal and single-price contingent orders.

Notice, however, that even if $\rho_{\epsilon}=0$, market makers still use the information contained in all the order flows when pricing an asset. Indeed, their demand can be written as $\mathbf{X}^{M M}(\boldsymbol{p})=\left(\boldsymbol{\Lambda}^{-1}-\right.$ A) $(\overline{\boldsymbol{v}}-\boldsymbol{p})$, and it is easy to see that the diagonality of $\boldsymbol{\Pi}_{\boldsymbol{\epsilon}}$ does not imply the diagonality of $\left(\boldsymbol{\Lambda}^{-1}-\mathbf{A}\right)$

\footnotetext{
${ }^{10}$ One can interpret the second term in the speculator's strategy 3.2 as a "correction" of the position the trader takes by only observing $s_{k j}$, due to the observation of $s_{k i}$. This correction is stronger (weaker) the higher (lower) is the correlation across error terms. Indeed, for a bivariate normal distribution, the value of $F \rho_{\epsilon}\left(\epsilon_{k 1}, \epsilon_{k 2}\right)$ is increasing in $\rho_{\epsilon}$ for all $\rho_{\epsilon} \in[-1,1]$ and all fixed $\left(\epsilon_{k 1}, \epsilon_{k 2}\right)$ : a higher correlation across error terms increases the probability that a joint bias in private signals occurs (see e.g. Tong, 1990).

${ }^{11}$ Notice that differently from Admati (1985) in this market, multicollinearity problems may determine the existence of a "Giffen" asset in the market makers' demand, but not in the demand of an informed trader (see Cespa, 2003).
} 


\section{The restricted system}

In the restricted system, a speculator $k$ can condition her demand for an asset $j$ on the whole vector of private signals $s_{k}$ and on the price of asset $j$ only. Assume she submits a demand schedule $X_{R k j}\left(s_{k}, p_{R j}\right)$, indicating the desired position in asset $j$ at every price $p_{R j}$, contingent on the available information. As done for the unrestricted system, I restrict my attention to linear equilibria. Therefore, equilibrium prices will be normally distributed. 12

The market makers of asset $j$ observe the asset order flow (that potentially carries information about both assets) but do not observe the order flow of the other asset. Formally, they thus observe $L_{R j}\left(p_{R j}\right)=\int_{0}^{1} X_{R k j}\left(s_{k}, p_{R j}\right) d k+u_{j}$. As the information structure is assumed to be ex-ante symmetric, demand functions and equilibria will again be symmetric. Suppose then that $X_{R k j}\left(\boldsymbol{s}_{k}, p_{R j}\right)=\boldsymbol{j}^{\prime} \mathbf{A}_{R} \boldsymbol{s}_{k}+\phi_{R j}\left(p_{R j}\right)$, where $\boldsymbol{j}$ is a column vector containing a 1 in the $j$-th position and a zero elsewhere, $\mathbf{A}_{R}$ is the matrix of trading intensities in the restricted system, and $\phi_{R j}(\cdot)$ is a linear function of the $j$-th price. The aggregate order flow of asset $j$ is then $L_{R j}\left(p_{R j}\right)=z_{R j}+\phi_{R j}\left(p_{R j}\right)$, where $z_{R j}=\boldsymbol{j}^{\prime}\left(\mathbf{A}_{R} \boldsymbol{v}+\boldsymbol{u}\right)$, denotes the order flow's informational content. Given competition and market makers' risk neutrality, the equilibrium price of asset $j$ is given by $p_{R j}=\bar{v}_{j}+\lambda_{R j} \boldsymbol{j}^{\prime}\left(\mathbf{A}_{R}(\boldsymbol{v}-\overline{\boldsymbol{v}})+\boldsymbol{u}\right)$, where $\lambda_{R j}=\left(\operatorname{Var}\left[z_{R j}\right]\right)^{-1} \operatorname{Cov}\left[v_{j}, z_{R j}\right]$, indicates the OLS regression coefficient of $v_{j}$ on $z_{R j}$ (i.e. the usual measure of market depth). Consequently, we have the following

Lemma 1 In every linear equilibrium of the restricted system, the vector of equilibrium prices is given by

$$
\boldsymbol{p}_{R}=\boldsymbol{\Lambda}_{R} \boldsymbol{z}_{R}+\left(I-\boldsymbol{\Lambda}_{R} \mathbf{A}_{R}\right) \overline{\boldsymbol{v}}
$$

\footnotetext{
${ }^{12}$ To the best of my knowledge, this is the first attempt to characterize the equilibrium in a multi-asset framework where competitive, risk averse traders receive different signals and bear restrictions in the number of asset prices they can condition upon.
} 
where $\boldsymbol{\Lambda}_{R}=\operatorname{diag}\left(\lambda_{R 1}, \lambda_{R 2}\right)$ and $\boldsymbol{z}_{R}=\mathbf{A}_{R} \boldsymbol{v}+\boldsymbol{u}$ are respectively the matrix of market depths and the vector of order flows' informational contents in the restricted model.

In the restricted system market makers can exploit cross-asset information in estimating an asset value if and only if speculators use both their signals when trading the asset. Conversely, in the unrestricted system even if $\mathbf{A}$ is diagonal, the price of an asset $j$ depends on the order flow of the other asset (to the extent that either $\Pi_{\boldsymbol{v}}$ or $\Pi_{\boldsymbol{u}}$ are not diagonal).

The following lemma characterizes informed speculators' equilibrium demand parameters.

Lemma 2 In every linear equilibrium of the restricted system, an informed speculator $k$ 's demand for asset $j=1,2$ is given by $X_{R k j}\left(\boldsymbol{s}_{k}, p_{R j}\right)=\boldsymbol{j}^{\prime} \mathbf{A}_{R}\left(\boldsymbol{s}_{k}-\overline{\boldsymbol{v}}\right)+b_{R j}\left(\bar{v}_{j}-p_{R j}\right)$, where

$$
\boldsymbol{j}^{\prime} \mathbf{A}_{R}=\gamma\left(\operatorname{Var}\left[v_{j} \mid \boldsymbol{s}_{k}, p_{R j}\right]\right)^{-1} \mathbf{c}_{2 j}, \text { and } b_{R j}=\gamma\left(\operatorname{Var}\left[v_{j} \mid \boldsymbol{s}_{k}, p_{R j}\right]\right)^{-1}\left(1-c_{1 j} / \lambda_{R j}\right)
$$

and $c_{1 j}, \mathbf{c}_{2 j}$, and $\operatorname{Var}\left[v_{j} \mid \boldsymbol{s}_{k}, p_{R j}\right]$ are defined in Appendix A.

Proof. See Appendix A.

The next proposition proves existence and uniqueness of the equilibrium in the restricted system, and the following corollary characterizes the equilibrium parameters.

Proposition 6 In the restricted system there exists a unique equilibrium in linear strategies. The equilibrium is symmetric and the price vector is given by (3.3), while the demand parameters are implicitly defined by (3.4).

Corollary 2 Let $a_{R j j}=\left(\mathbf{A}_{R}\right)_{j j}$ and $a_{R j i}=\left(\mathbf{A}_{R}\right)_{j i}$. In the unique linear equilibrium of the restricted system: 
1. $a_{R j i}>0$ if and only if $\rho_{\epsilon} \sqrt{\tau_{\epsilon_{j}} / \tau_{\epsilon_{i}}}<\rho_{v} \sqrt{\tau_{v_{j}} / \tau_{v_{i}}}$;

2. (a) $a_{R j j}=\gamma \tau_{\epsilon_{j}}\left(1-\gamma^{-1} a_{R j i} \operatorname{Cov}\left[\epsilon_{1}, \epsilon_{2}\right]\right)>0$ and (b) $\lambda_{R j}>0$;

3. if $\rho_{\epsilon}=0, a_{R j j}=\gamma \tau_{\epsilon_{j}}$ and $a_{R j i} \neq 0$;

4. if $\rho_{\epsilon} \sqrt{\tau_{\epsilon_{j}} / \tau_{\epsilon_{i}}}=\rho_{v} \sqrt{\tau_{v_{j}} / \tau_{v_{i}}}, a_{R j j}=\gamma \tau_{\epsilon_{j}}, a_{R j i}=0$, and $b_{R j}=-a_{R j j}$.

Proof. See Appendix A.

The interpretation of these results is as follows. For part 1, suppose an informed speculator trading asset 1 receives two "high" signals $s_{k 1}, s_{k 2}$. This may be the effect of either fundamental information, or of errors in the signals. The first possibility is more likely the stronger is the correlation of asset payoffs compared to error terms' correlation and the higher is the relative dispersion of asset payoffs compared to error terms' relative dispersion. In this case, indeed, the effect of fundamental information dominates the effect of errors in the signal vector. For part 2 (a) suppose that $a_{R 12}>0$. This means that an informed trader increases her speculative position in asset 1 upon receiving "good news" about asset 2. However, if $\rho_{\epsilon}>0$, good news about asset 1 may come from the joint effect of signal error terms. Therefore, the trader scales down the weight she puts on $s_{k 1}$, the higher is the trading intensity she puts on $s_{k 2}$. For 2 (b), the impossibility of observing more than one order flow when pricing an asset eliminates the multicollinearity effects that occur in the unrestricted system. Therefore, the matrix $\boldsymbol{\Lambda}_{R}$ is positive definite. ${ }^{13}$ For part 3, the intuition is that a given signal $s_{k i}$ is useful in trading an asset $j \neq i$ if it carries information either about $v_{j}$ or about the error term $\epsilon_{k j}$. As the correlation across error terms vanishes, $s_{k i}$ is still useful for the information it contains about $v_{j}$. Therefore, speculators use it in trading asset $j$. Notice that this result is

\footnotetext{
${ }^{13}$ This explanation is therefore different from the one in Caballé and Krishnan (1994), where the phenomenon is due to the hypothesis of imperfect competition among insiders that prevents the existence of unexploited arbitrage opportunities.
} 
in stark contrast with corollary 1 market makers' inability to observe all order flows renders both signals useful to informed traders beyond the correlated information about error terms they contain. The last result is not surprising given what we said above. If $\rho_{\epsilon} \sqrt{\tau_{\epsilon_{j}} / \tau_{\epsilon_{i}}}=\rho_{v} \sqrt{\tau_{v_{j}} / \tau_{v_{i}}}$, there is no way for a speculator to disentangle error terms from information by pooling the two signals she receives. As a consequence $a_{R j i}=0$.

Remark 3 As done for the unrestricted system, let us consider more closely a trader's strategy in the restricted system:

$$
X_{R k j}\left(s_{k}, p_{R j}\right)=a_{R j j}\left(s_{k j}-\bar{v}_{j}\right)+a_{R j i}\left(s_{k i}-\bar{v}_{i}\right)+b_{R j}\left(\bar{v}_{j}-p_{R j}\right) .
$$

Again, $k$ 's trading intensity in asset $j$ is the composition of 2 effects: a direct one stemming from the informational advantage the speculator has over the rest of the market in asset $j$, and an indirect one coming from the informational advantage she has on the other asset, to the extent that she received conditionally correlated signals. Suppose $\rho_{\epsilon} \sqrt{\tau_{\epsilon_{j}} / \tau_{\epsilon_{i}}}<\rho_{v} \sqrt{\tau_{v_{j}} / \tau_{v_{i}}}$, and that $s_{k j}>\bar{v}_{j}, s_{k i}>\bar{v}_{i}$. As the effect of fundamental information dominates the effect of errors in the signal vector, the speculator reinforces her belief that the asset value is high and increases her long position. If $\bar{v}_{j}>p_{R j}$, such a long position is further increased because of the low price the market gives to the asset. 14

\footnotetext{
${ }^{14}$ Numerical simulations show that $b_{R j}>0$.
} 


\section{Comparing the unrestricted with the restricted system}

\section{Trading aggressiveness and price informativeness}

In section 2, I have related a trader's aggressiveness to the type of order she submits in a single asset market, and analyzed the implications of different order types for price informativeness. In this section, I first show that in a multi-asset world not only the type of order, but also the way prices are formed influences a trader's aggressiveness. I then analyze the relationship between trading aggressiveness and price informativeness.

Proposition 7 Let $a_{j j}=(\mathbf{A})_{j j}$ and $a_{j i}=(\mathbf{A})_{j i}$. Then,

1. when $\rho_{v}=0$ and $\rho_{\epsilon} \neq 0, a_{j j}>a_{R j j},\left|a_{j i}\right|>\left|a_{R j i}\right|$;

2. when $\rho_{\epsilon}=0$ and $\rho_{v} \neq 0, a_{j j}=a_{R j j},\left|a_{j i}\right|<\left|a_{R j i}\right|$;

3. when $\rho_{\epsilon}=\rho_{v}=0$ and $\rho_{u} \neq 0, a_{j j}=a_{R j j}=\tau_{\epsilon_{j}},\left|a_{j i}\right|=\left|a_{R j i}\right|=0$.

Proof. See Appendix A.

Proposition 7 shows that the ranking of traders' aggressiveness across the two systems depends on correlation coefficients. As shown in corollaries 1 and 2 informed speculators combine private signals to disentangle error terms from fundamental information. When $\rho_{v}=0$ and $\rho_{\epsilon} \neq 0$, in the unrestricted system this can be done comparing signals with prices whereas in the restricted system traders compare signals with prior means. As prices are better estimators of the fundamentals, traders in the unrestricted system are better able to assess the extent of their signal bias. As a consequence, they speculate more aggressively on their private information. When $\rho_{\epsilon}=0$ and $\rho_{v} \neq 0$, in the unrestricted system informed traders don't use multidimensional private information to improve their strategies, and submit single signal-contingent orders. Conversely, in the restricted 
system they use the cross asset information contained in their signals (and not already fully reflected in the price). This, in turn, boosts their trading aggressiveness. Finally, when $\rho_{\epsilon}=\rho_{v}=0$ and $\rho_{u} \neq 0$ in both systems there is no way for speculators to disentangle error terms from information, and their trading aggressiveness coincide. 15

Price informativeness is measured by the reduction in the unconditional variance of an asset $j$ 's payoff due to the observation of the vector of order flows. Thus, in the unrestricted system $I_{p_{j}}=\tau_{v_{j}}^{-1}-\operatorname{Var}\left[v_{j} \mid \boldsymbol{z}\right]$, while in the restricted system $I_{p_{R j}}=\tau_{v_{j}}^{-1}-\operatorname{Var}\left[v_{j} \mid \boldsymbol{z}_{R}\right]$. This definition is natural in the unrestricted system as it corresponds to the ex-ante volatility of asset $j$ 's price. In the restricted system it captures the point of view of an econometrician interested in estimating the deep parameters of the market, that regresses asset $j$ 's fundamentals on the order flows, and measures the informativeness of these regressors using $I_{p_{R j}}$. Alternatively, it captures the perspective of a trader who, before submitting an order observes the past asset price as well as the price formed in a related market. I will thus say that the unrestricted system prices are more informative than those of the restricted system if and only if $I_{p_{j}} \geq I_{p_{R j}}$, for $j=1,2.16$

Straightforward normal calculations give

$$
\begin{aligned}
I_{p_{j}} & =\tau_{v_{j}}^{-1}\left(\frac{\rho_{v_{j}, z_{j}}^{2}+\rho_{v_{j}, z_{i}}^{2}-2 \rho_{z_{j}, z_{i}} \rho_{v_{j}, z_{j}} \rho_{v_{j}, z_{i}}}{1-\rho_{z_{j}, z_{i}}^{2}}\right), \\
I_{p_{R j}} & =\tau_{v_{j}}^{-1}\left(\frac{\rho_{v_{j}, z_{R j}}^{2}+\rho_{v_{j}, z_{R i}}^{2}-2 \rho_{z_{R j}, z_{R i}} \rho_{v_{j}, z_{R j}} \rho_{v_{j}, z_{R i}}}{1-\rho_{z_{R j}, z_{R i}}^{2}}\right) .
\end{aligned}
$$

\footnotetext{
${ }^{15}$ Part 2 of proposition 7 may seem to contrast with the intuition formed in section 2. As in the restricted system strategies do not depend on all the information related to the fundamentals - as in the market order market - one may think that a trader should also speculate less aggressively. However, in the restricted system market makers do not observe both order flows; thus, lack of cross-conditioning ability does not expose traders to price movements spurred by events affecting other order-flows.

${ }^{16}$ For an efficiency comparison in a one-asset, strategic set up where traders have information both on the fundamental value and on the source of noise see Palomino (2001).
} 
It is useful to compare the above formulas with their analogues in a single asset framework (equation (2.1)). Differently from the one-asset setup - for a given payoff volatility - in a multi-asset market price informativeness depends on two factors: the total correlation between order flows and the asset payoff - taking into account the potential "correction" for redundant information - and the correlation across order-flows. 17 The more correlated with the fundamentals the order flows are (i.e. the higher is the numerator in each one of (4.5)), the more fundamental information can be extracted by observing them. The more correlated among themselves the order flows are (i.e. the lower is the denominator in each one of (4.5)), the easier it is to disentangle noise from information within each order flow. 18

From now on I restrict attention to the "homoscedastic case," assuming that $\tau_{v_{j}}=\tau_{v}, \tau_{u_{j}}=\tau_{u}$, and that $\tau_{\epsilon_{j}}=\tau_{\epsilon}$, for $j=1,2$. Besides simplifying the analysis, this also allows us to concentrate on correlation effects, abstracting from the role that differences in signals' precisions, payoffs dispersions and noise trader demands' volatilities play on the use of private information.

Proposition 8 In the homoscedastic case, when $\rho_{\epsilon}=\rho_{u}=0$, for $\rho_{v}$ small, prices in the restricted system are more informative than in the unrestricted system.

Proof. See Appendix A.

Numerical simulations support the above result also for larger values of $\left|\rho_{v}\right|$. In particular, letting $\rho_{v} \in\{-.9,-.8, \ldots, .8, .9\}, \rho_{u}=\rho_{\epsilon}=0$ and $\gamma, \tau_{u}, \tau_{v}, \tau_{\epsilon} \in\{.2, .4, .5, .6, .8,1,3,4\}$

\footnotetext{
${ }^{17}$ To understand the negative term in the numerator of (4.5), consider for instance the case in which $\rho_{v_{j}, z_{R j}}>0$, $\rho_{v_{j}, z_{R i}}>0$, and $\rho_{z_{R j}, z_{R i}}>0$. In this situation, observing a high value of $z_{R j}$ induces one to believe that $v_{j}$ is high. This belief is further reinforced if $z_{R i}$ is also high. However, as $\rho_{z_{R j}, z_{R i}}>0$, such inference could be upward biased as part of the $z_{R j}$ and $z_{R i}$ realizations may be due to the positive correlation that links these two random variables. The negative term in the numerator of (4.5) corrects for this kind of problems.

${ }^{18}$ For example, observing two "large" order flows realizations, and knowing that order flows are, say, positively correlated only through fundamentals, leads one to conclude that these signals are likely to be result of high fundamentals rather than positive noise traders demands.
} 
price informativeness is always higher in the restricted system (see figures 1 and 2, panel (a) for an example). 19

According to proposition 7 if order flows are correlated only through payoffs, traders in the restricted system speculate more aggressively than in the unrestricted system. As a result, the correlation between each order flow and the asset payoff and across order flows in the restricted system is larger than in the unrestricted system. This has two effects on price informativeness. First, owing to the correction for redundant information, it may make the total correlation between order flows and the asset payoff in the restricted system lower than in the unrestricted system. Second, it unambiguously improves the ability to disentangle noise from information within each order flow in the restricted system. As the latter effect is always stronger than the former, prices end up being more informative in the restricted system. 20

This finding is in stark contrast with the common wisdom that a major benefit of a multi-price contingent system is that of rendering the market more efficient. Indeed, Amihud and Mendelson (1991b, p. 127) argue that a "mechanism which enables simultaneous conditioning of orders for different assets (...) would increase the information available to traders, improve value discovery and reduce volatility." This assertion points at the positive effect that observing multiple sources of correlated information has. By contrast, proposition 8 unveils the dark side of a multi-price contingent system, by uncovering its feedback effect on price informativeness.

The above result can also be interpreted as a multi-asset analogue of the Grossman and Stiglitz (1980) paradox on the impossibility of informationally efficient markets. Indeed, the more information is revealed by prices (and the more prices traders observe), the lower is the weight traders put

\footnotetext{
${ }^{19}$ Simulations were run with the aid of Octave.

${ }^{20}$ Notice that the condition given in proposition 8 is sufficient but it is by no means a necessary one. It is easy to show that in the homoscedastic case when $\rho_{v}=\rho_{\epsilon} \neq 0$ and $\rho_{u}=0$, prices in the restricted system are more informative than in the unrestricted system.
} 
on their signals; this, in turn, makes prices in the unrestricted system less informative than in the restricted system. It is however worth stressing that in a in a multi-asset setup a stronger aggressiveness per-se does not grant a higher price informativeness; for as a result of a strong aggressiveness, order flows can be highly correlated among themselves but poorly correlated with the fundamentals (examples can be constructed where for $\rho_{\epsilon} \neq 0$ and $\rho_{v}=\rho_{u}=0$ in the homoscedastic case, prices in the restricted system are more informative than in the unrestricted system, although traders speculate more aggressively in the unrestricted system).

How reasonable is the chosen parameterization and how robust are the results presented above? First of all, it seems realistic to assume that $\rho_{\epsilon}=0$, as it is likely that each signal in the vector a trader receives contains information produced by a different analyst. More interesting is the situation in which noise traders' demands are correlated. In this case, the final effect on price informativeness is ambiguous. For values of $\left|\rho_{u}\right|$ smaller than $.002 \%$, and for the same parameter space described above, the restricted system prices are still more informative than those of the unrestricted system. However, larger values of $\left|\rho_{u}\right|$ reduce the strong correlation across order flows in the restricted system (i.e. increase the denominator of $I_{p_{R j}}$ in (4.5)), dampening its positive effect on price informativeness, and, for some parameter values, revert the informativeness ranking.

Please insert figure 1 here.

\section{Informed expected utility and noise traders' losses}

In this section I study traders' welfare in the two systems. As to noise traders, their expected losses depend on the price impact of trades, i.e. the extent to which prices move as a result of market 
makers' order flow observation. For what concerns informed traders, the decision to trade in the unrestricted instead of the restricted system depends on the informational advantage they are able to retain vis-à-vis market makers in each mechanism.

To fix notation, indicate with $\pi_{k}=\boldsymbol{x}_{k}^{\prime}(\boldsymbol{v}-\boldsymbol{p})$ and with $\pi_{R k}=\boldsymbol{x}_{R k}^{\prime}\left(\boldsymbol{v}-\boldsymbol{p}_{R}\right)$ respectively an informed trader $k$ 's profit in the unrestricted and in the restricted system. Also let $-E\left[\boldsymbol{u}^{\prime}(\boldsymbol{v}-\boldsymbol{p})\right]=$ $\operatorname{tr}\left(\boldsymbol{\Lambda} \boldsymbol{\Pi}_{\boldsymbol{u}}^{-1}\right)$ and $-E\left[\boldsymbol{u}^{\prime}\left(\boldsymbol{v}-\boldsymbol{p}_{R}\right)\right]=\operatorname{tr}\left(\boldsymbol{\Lambda}_{R} \boldsymbol{\Pi}_{\boldsymbol{u}}^{-1}\right)$ denote respectively noise traders' expected losses in the unrestricted and in the restricted system.

As for informed traders, in the unrestricted system a straightforward application of lemma B1 gives $E\left[-\exp \left\{-\gamma^{-1} \pi_{k}\right\}\right]=-|\boldsymbol{\Pi}|^{1 / 2}\left|\boldsymbol{\Pi}+\boldsymbol{\Pi}_{\boldsymbol{\epsilon}}\right|^{-1 / 2}$, whereas for the restricted system see Appendix B.

Proposition 9 In the homoscedastic case, when $\rho_{\epsilon}=\rho_{u}=0$, for $\rho_{v}$ small, noise traders' expected losses are always higher in the restricted system.

\section{Proof. See Appendix A.}

With the above parameter configuration, speculators trade more aggressively in the restricted system, embedding more information in the order flows. This worsens market makers' adverse selection problem in the restricted system, making the price impact of trade stronger and noise traders' expected losses higher.

Numerical simulations support the result also for higher values of $\left|\rho_{v}\right|$. In particular, using the same parameterization of section 4, noise traders' expected losses are always higher in the restricted system (see figures 1 and 2, panel (b) for an example). 21

\footnotetext{
${ }^{21}$ If noise traders' demands are correlated the above ranking may be reverted. To see why, notice that in the homoscedastic case $-E\left[\boldsymbol{u}^{\prime}(\boldsymbol{v}-\boldsymbol{p})\right]=2 \tau_{u}^{-1}\left(\lambda_{1}+\rho_{u} \lambda_{2}\right)$, and $-E\left[\boldsymbol{u}^{\prime}\left(\boldsymbol{v}-\boldsymbol{p}_{R}\right)\right]=2 \tau_{u}^{-1} \lambda_{R}$, where $\lambda_{1}$ and $\lambda_{2}$ indicate respectively the main and off-diagonal terms of the matrix $\Lambda$. Thus, in the unrestricted system as the price of each asset reacts to both order-flows, noise traders' losses also depend on the off-diagonal terms of the matrix $\boldsymbol{\Lambda}$. Conversely, in the
} 
For what concerns informed speculators, as long as their risk aversion is strong and the quality of private information is poor (e.g. $\gamma$ and $\tau_{\epsilon}$, smaller than 1) they are better off in the unrestricted system but as $\tau_{\epsilon}$ or $\gamma$ increase, they are better off in the restricted system (see figures 1 and 2, panel (c) for an example). The intuition is along the lines of what was said in section 2. Traders prefer the unrestricted system as long as they speculate less aggressively, (either because their information is poor, or because their risk aversion is high). In this situation they take advantage of multiple sources of information (prices) without losing much of their advantage vis-à-vis market makers. However, as their information becomes more precise (or they are less averse to the risk of trading), their aggressiveness increases and the possibility for market makers to observe both order-flows in the unrestricted system becomes a drawback. In this case, thus, they rather trade on the restricted system where - although they impound more correlated information in each price - market makers only observe the order flow of the asset they price. 22

Please insert figure 2 here.

restricted system, noise traders' losses in each asset only depend on the price impact of trades in the relative market. For small values of $\left|\rho_{u}\right|$ (e.g. $\left|\rho_{u}\right| \leq .0001$ ), cross-order flows effects are mild and noise traders are always better off in the unrestricted system. As $\left|\rho_{u}\right|$ increases, however, the reverse may happen.

${ }^{22}$ More precisely, the advantage of trading in the unrestricted system is stronger, the more concentrated is noise traders' demand, and the better is the ex-ante information about fundamentals. In this case, indeed, for high values of $\gamma$ and $\tau_{\epsilon}$, the stronger aggressiveness displayed in the use of multidimensional information in the restricted system counteracts market makers' possibility to observe multiple order flows in the unrestricted system. 


\section{Discussion and extensions}

\section{An alternative information structure}

In the analysis conducted so far, I have ruled out the possibility that traders' private signals are biased by the presence of a "common" error term. However, insofar as signals may incorporate an industry bias, such a possibility becomes relevant.

Formally, in this case a trader $k$ 's private signal (vector) is given by $\boldsymbol{s}_{k}=\boldsymbol{v}+\boldsymbol{\eta}+\boldsymbol{\epsilon}_{k}$, where $\boldsymbol{\eta} \sim N\left(\mathbf{0}, \boldsymbol{\Pi}_{\boldsymbol{\eta}}^{-1}\right)$, represents the common error term that I assume to be independent from both the payoff $(\operatorname{Cov}[\boldsymbol{\eta}, \boldsymbol{v}]=\mathbf{0})$, and the idiosyncratic component $\left(\operatorname{Cov}\left[\boldsymbol{\eta}, \boldsymbol{\epsilon}_{k}\right]=\mathbf{0}, \forall k \in[0,1]\right)$. An immediate consequence of this assumption is that in this market the vector of average private signals no longer reveals the true asset payoffs. This increases traders' uncertainty in both systems, potentially affecting their trading aggressiveness and thus price efficiency. 23

To what extent do the results obtained in section 4 carry over to the present setup? While a closed form solution ceases to be available for the unrestricted system, numerical methods can be used to compute the linear equilibria of both systems in the homoscedastic case. The results broadly confirm most of the intuitions gained in the previous sections. In particular, it is still true that in the presence of correlation only across payoffs traders use multidimensional private information in the restricted system but refrain from doing so in the unrestricted system. This, in turn, increases the correlation across order flows in the restricted system, making its prices more informative than those of the unrestricted system. Results on noise traders' expected losses are, however, inconclusive: for large values of $\gamma, \tau_{u}, \tau_{\epsilon}$, and $\tau_{\eta}$, these are higher in the unrestricted system; the opposite occurs for

\footnotetext{
${ }^{23}$ In a one-asset, limit order setup where traders' signals are biased by a common and an idiosyncratic error component, it is easy to verify that the unique linear equilibrium trading aggressiveness is given by $a=\left(\tau_{\epsilon}+\tau_{\eta}+a^{2} \tau_{u}\right)^{-1} \gamma \tau_{\epsilon} \tau_{\eta}$, where $a$ is the unique positive root of the cubic $F(a)=a^{3} \tau_{u}+a\left(\tau_{\epsilon}+\tau_{\eta}\right)-\gamma \tau_{\epsilon} \tau_{\eta}=0$, and $\tau_{\eta}$ is the precision of the common error term. As one can verify $a<\gamma \tau_{\epsilon}$. Thus, the higher uncertainty makes traders scale back their aggressiveness.
} 
smaller values of the above parameters. 24

\section{An "Intermediate" system}

The results obtained in the previous sections have shown that a mechanism disseminating a large amount of (endogenous) public information may have a negative impact on price informativeness. If this is the case, a system where market makers observe both order flows, while informed speculators bear single-price restrictions, should deliver prices that are contemporaneously less informative than those of the restricted system, and more informative than those of the unrestricted system. The opening call auction of the NYSE provides an example of such a system. There, each specialist handles more than one stock, and can thus make "cross" asset inference at the moment of setting the opening price; speculators, however, condition their strategies only on the price of the stock they want to trade. 25

Notice that differently from the restricted system, in this case market makers learn cross asset information independently from informed traders' equilibrium behavior. As a consequence, the equilibrium price of each asset is informationally equivalent to the linear combination of both order flows' informational content. In this framework, a closed form solution is unavailable. However, restricting attention to the homoscedastic case, it can be shown that a linear rational expectations equilibrium exists. 26

To compare price efficiencies, I run simulations on the three models, using the same parameterization of section 4 . The results broadly accord to intuition: for most parameter values, when only

\footnotetext{
${ }^{24}$ Computations are available from the author. Numerical simulations where run letting $\rho_{v} \in\{-.9,-.8, \ldots, .8, .9\}$, $\rho_{u}=\rho_{\epsilon}=\rho_{\eta}=0$, and $\gamma, \tau_{u}, \tau_{v}, \tau_{\epsilon}, \tau_{\eta} \in\{.2, .4, .5, .6, .8,1,3,4\}$. For this parameters' space, increased payoff uncertainty has a different impact on traders' aggressiveness across the two systems: if $\rho_{\epsilon}=\rho_{u}=\rho_{\eta}=0$ and $\rho_{v} \neq 0$, numerical simulations show that $a_{j j}>0, a_{R j j}>0$, and $a_{j j}>a_{R j j}$, while $\left|a_{R j i}\right|>\left|a_{j i}\right|=0$.

${ }^{25}$ Lindsay and Schaede (1990, p. 12) report that in 1987 “(...) the average number was 3.7 stocks per specialist.”

${ }^{26}$ The proof is available from the author. Uniqueness of the equilibrium is an issue. Numerical simulations have been carried out and for different initial conditions the solution of the fixed point problem did not change.
} 
correlation across fundamentals affects order flows, speculators in the restricted system trade more aggressively than in the intermediate system; in turn speculators in the intermediate system trade more aggressively than in the unrestricted system. This induces a price vector in the intermediate system that on the one hand is less informative than in the restricted system and on the other hand is more informative than in the unrestricted system (figure 3, panel (c)). 27

There are however exceptions: when (a) noise traders' demand is very dispersed $\left(\tau_{u} \leq .2\right)$, and (b) correlation across payoffs is strong $\left(\left|\rho_{v}\right| \geq .8\right)$ the aggressiveness-informativeness ranking between the restricted and the intermediate system is reversed. Owing to high noise traders' demand dispersion, risk-averse speculators in the restricted system suffer from a large conditional volatility of the payoff and scale back their aggressiveness. Conversely, in the intermediate system, market makers' multiple order flows observation dampens the price impact of trades reducing speculators' payoff conditional volatility. As a result, speculators trade more aggressively and embed more information in the order flows rendering prices more informative (figure 3, panel (a)).

Results for noise traders' expected losses are inconclusive: for some parameterizations noise traders are better off in the intermediate system than in the unrestricted one (figure 3, panel (b)) while for other parameterizations the reverse occurs (figure 3, panel (d)). 28

Please insert figure 3 here.

\footnotetext{
${ }^{27}$ More precisely, the aggressiveness ranking makes the total correlation between order flows and the asset payoff and across order flows in the restricted system larger than in the intermediate system. As a consequence, the restricted system price vector turns out to be more informative than the intermediate system one. As for the intermediate-unrestricted informativeness ranking, the pattern parallels what has been observed in section 4 .

${ }^{28}$ To study price informativeness, simulations have been extended letting $\gamma, \tau_{v}, \tau_{u}$ and $\tau_{\epsilon} \in\{.01, .1, .2, .4, .5, .6$, $.8,1,3,4\}$. As far as noise traders' expected losses, in some simulations they can even be higher in the intermediate system than in the restricted one.
} 


\section{Conclusions}

Advances in information technology are deeply modifying the way stock market procedures are handled. ITG, a technology company, through its trading platform QuantEX permits a submission strategy ("Pairs") that automatically executes orders "when the spread differential between two stocks reaches a specified level." The Optimark platform provides a system allowing traders to specify different parameters upon which to condition execution and Bondconnect implements a mechanism allowing the exchange of portfolios of assets. These examples testify the effort to improve trade execution, allowing more flexibility both in the determination of the number of assets to exchange and in the amount of trade relevant information to exploit when submitting an order. Motivated by this evidence, I have analyzed two trading systems where competitive speculators exploit multi-dimensional sources of private information, and contrasted their properties on the basis of two different pricing schemes. In the unrestricted mechanism, traders submit multi-price contingent demand functions and market makers set prices observing all order flows; in the restricted mechanism, speculators submit standard limit orders and market makers bear a single order flow restriction.

The results show that the way traders use private information crucially depends both on the type of order they submit and on the specific price formation mechanism one considers. Indeed, to the extent that private and public information are substitutable, a system allowing traders to observe more public signals, under some conditions, reduces the weight they put on their private signals. This, in turn, reduces the amount of information embedded in the order flows and may ultimately make a multi-price contingent mechanism less efficient than a single price contingent one, in stark contrast with the view that a mechanism of the first type should render prices more informative. The paper thus uncovers the existence of a possible trade-off between the quantity of multi-dimensional 
public information that traders can access, and its resulting quality.

Many issues are left for future research. In particular, a dynamic extension of the model presented here would allow one to study how information updating through the observation of past prices influences traders' behavior and market properties. 29 Also, introducing production in the restricted model would allow to study the interactions among firms' competition, traders' behavior, and stock price determination. This last issue seems particularly relevant given that there is virtually no analysis of the links between firms' conduct in the product market and investors' reactions to the resulting stock price effects. 30

\footnotetext{
${ }^{29}$ See He and Wang (1995), Vives (1995a;1995b), and Cespa (2002) for models of single-asset, dynamic trading in a competitive stock market. Chan (1992) studies price determination in a multi-asset Kyle (1985) market where in each period $n$, market makers observe the order flow of the asset they price and the period $n-1$ prices of all the other assets. However, in his case informed speculators' behavior is not modeled, thus the feedback effects of prices on private information usage cannot be analyzed.

${ }^{30}$ Fishman and Hagerty (1989) and Dow and Rahi (2002) analyze how the information gathered in the market place affects a firm's investment decisions; Gertner, Gibbons, and Scharfstein (1988) investigate how product-market considerations influence an informed firm's decision to reveal information to the capital market; Poitevin (1989) shows how a financially-constrained entrant, by signaling information about its leverage to the capital market, spurs a "deep-pocket" incumbent to engage in predatory practices.
} 


\section{Appendix A}

Proof of proposition 5. Notice that in every linear equilibrium, as $\boldsymbol{p}=E[\boldsymbol{v} \mid \boldsymbol{z}]=\boldsymbol{\Pi}^{-1}\left(\boldsymbol{\Pi}_{\boldsymbol{v}} \overline{\boldsymbol{v}}+\right.$ $\left.\mathbf{A}^{\prime} \Pi_{\boldsymbol{u}} \boldsymbol{z}\right)$, where $(\operatorname{Var}[\boldsymbol{v} \mid \boldsymbol{z}])^{-1}=\boldsymbol{\Pi}=\boldsymbol{\Pi}_{\boldsymbol{v}}+\mathbf{A}^{\prime} \boldsymbol{\Pi}_{\boldsymbol{u}} \mathbf{A}$, the vector of equilibrium prices is observationally equivalent to $z$. Next, owing to CARA preferences, the trader's demand vector is given by $\mathbf{X}_{k}\left(\boldsymbol{s}_{k}, \boldsymbol{p}\right)=\left(\operatorname{Var}\left[\boldsymbol{v} \mid \boldsymbol{s}_{k}, \boldsymbol{p}\right]\right)^{-1}\left(E\left[\boldsymbol{v} \mid \boldsymbol{s}_{k}, \boldsymbol{p}\right]-\boldsymbol{p}\right)$. Assume that the matrix $\mathbf{A}$ is invertible, then $\mathbf{A}^{-1} \boldsymbol{z}=\left(\mathbf{A}^{\prime} \boldsymbol{\Pi}_{\boldsymbol{u}} \mathbf{A}\right)^{-1}\left(\boldsymbol{\Pi} \boldsymbol{p}-\boldsymbol{\Pi}_{\boldsymbol{v}} \overline{\boldsymbol{v}}\right) \mid \boldsymbol{v} \sim N\left(\boldsymbol{v}, \mathbf{A}^{-1} \boldsymbol{\Pi}_{\boldsymbol{u}}^{-1}\left(\mathbf{A}^{-1}\right)^{\prime}\right)$, and $\boldsymbol{s}_{k} \mid \boldsymbol{v} \sim N\left(\boldsymbol{v}, \boldsymbol{\Pi}_{\boldsymbol{\epsilon}}^{-1}\right)$. Thus, using the properties of the multivariate normal, $\left(\operatorname{Var}\left[\boldsymbol{v} \mid \boldsymbol{s}_{k}, \boldsymbol{p}\right]\right)^{-1}=\boldsymbol{\Pi}_{\boldsymbol{v}}+\mathbf{A}^{\prime} \boldsymbol{\Pi}_{\boldsymbol{u}} \mathbf{A}+\boldsymbol{\Pi}_{\boldsymbol{\epsilon}}$ $=\boldsymbol{\Pi}+\boldsymbol{\Pi}_{\boldsymbol{\epsilon}}$ and $E\left[\boldsymbol{v} \mid \boldsymbol{s}_{k}, \boldsymbol{p}\right]=\left(\boldsymbol{\Pi}+\boldsymbol{\Pi}_{\boldsymbol{\epsilon}}\right)^{-1}\left(\boldsymbol{\Pi} E[\boldsymbol{v} \mid \boldsymbol{z}]+\boldsymbol{\Pi}_{\boldsymbol{\epsilon}} \boldsymbol{s}_{k}\right)$ (see for instance DeGroot, 1969). Plugging these expressions into the trader's strategy and simplifying $\mathbf{X}_{k}\left(\boldsymbol{s}_{k}, \boldsymbol{p}\right)=\gamma \boldsymbol{\Pi}_{\boldsymbol{\epsilon}}\left(\boldsymbol{s}_{k}-\boldsymbol{p}\right)$. Hence, $\mathbf{A}=\gamma \boldsymbol{\Pi}_{\boldsymbol{\epsilon}}$ is a positive definite matrix.

Q.E.D.

Proof of lemma 2 In the restricted system a speculator $k$ determines her position in each asset separately and independently. Therefore, owing to CARA preferences, her demand for each asset $j$ is given by $X_{R k j}\left(p_{R j}, \boldsymbol{s}_{k}\right)=\gamma\left(\operatorname{Var}\left[v_{j} \mid p_{R j}, \boldsymbol{s}_{k}\right]\right)^{-1}\left(E\left[v_{j} \mid p_{R j}, \boldsymbol{s}_{k}\right]-p_{R j}\right)$, and because of the assumed ex-ante symmetric information structure (whereby each informed trader receives a signal of the same precision), demand functions and equilibria will be symmetric. Then, $E\left[v_{j} \mid p_{R j}, \boldsymbol{s}_{k}\right]=$ $\bar{v}_{j}+\left(c_{1 j} \mathbf{c}_{2 j}^{\prime}\right)\left(\left(\boldsymbol{j}^{\prime} \lambda_{j}^{-1}(\boldsymbol{p}-\overline{\boldsymbol{v}})\right)^{\prime}\left(\boldsymbol{s}_{k}-\overline{\boldsymbol{v}}\right)^{\prime}\right)^{\prime}$, where the scalar $c_{1 j}$ and the vector $\mathbf{c}_{2 j}^{\prime}$ are defined as follows: $\left(c_{1 j} \mathbf{c}_{2 j}^{\prime}\right) \operatorname{Var}\left[p_{R j}, \boldsymbol{s}_{k}\right]=\operatorname{Cov}\left[v_{j},\left\{p_{R j}, \boldsymbol{s}_{k}\right\}\right]$. Standard normal computations give

$$
\operatorname{Var}\left[p_{R j}, s_{k}\right]=\left(\begin{array}{cc}
\boldsymbol{j}^{\prime}\left(\mathbf{A}_{R} \boldsymbol{\Pi}_{\boldsymbol{v}}^{-1} \mathbf{A}_{R}^{\prime}+\boldsymbol{\Pi}_{\boldsymbol{u}}^{-1}\right) \boldsymbol{j} & \boldsymbol{j}^{\prime} \mathbf{A}_{R} \boldsymbol{\Pi}_{\boldsymbol{v}}^{-1} \\
\left(\boldsymbol{j}^{\prime} \mathbf{A}_{R} \boldsymbol{\Pi}_{\boldsymbol{v}}^{-1}\right)^{\prime} & \boldsymbol{\Pi}_{\boldsymbol{v}}^{-1}+\boldsymbol{\Pi}_{\boldsymbol{\epsilon}}^{-1}
\end{array}\right)
$$

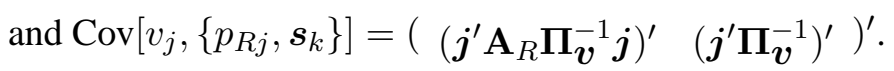


Inverting (A1) I obtain

$$
\left(\operatorname{Var}\left[p_{R j}, \boldsymbol{s}_{k}\right]\right)^{-1}=\left(\begin{array}{cc}
D_{1}^{-1} & -D_{1}^{-1} \boldsymbol{j}^{\prime} \mathbf{A}_{R}\left(\boldsymbol{\Pi}_{\boldsymbol{v}}+\boldsymbol{\Pi}_{\boldsymbol{\epsilon}}\right)^{-1} \boldsymbol{\Pi}_{\boldsymbol{\epsilon}} \\
-D_{1}^{-1}\left(\boldsymbol{j}^{\prime} \mathbf{A}_{R}\left(\boldsymbol{\Pi} \boldsymbol{v}+\boldsymbol{\Pi}_{\boldsymbol{\epsilon}}\right)^{-1} \boldsymbol{\Pi}_{\boldsymbol{\epsilon}}\right)^{\prime} & D_{2}
\end{array}\right)
$$

where $D_{1}=\boldsymbol{j}^{\prime}\left(\mathbf{A}_{R}\left(\boldsymbol{\Pi}_{\boldsymbol{v}}+\boldsymbol{\Pi}_{\boldsymbol{\epsilon}}\right)^{-1} \mathbf{A}_{R}^{\prime}+\boldsymbol{\Pi}_{\boldsymbol{u}}^{-1}\right) \boldsymbol{j}$, and

$$
D_{2}=\boldsymbol{\Pi}_{\boldsymbol{v}}\left(\boldsymbol{\Pi}_{\boldsymbol{v}}+\boldsymbol{\Pi}_{\boldsymbol{\epsilon}}\right)^{-1} \boldsymbol{\Pi}_{\boldsymbol{\epsilon}}+D_{1}^{-1}\left(\boldsymbol{\Pi}_{\boldsymbol{v}}^{-1}+\boldsymbol{\Pi}_{\boldsymbol{\epsilon}}^{-1}\right)^{-1} \boldsymbol{\Pi}_{\boldsymbol{v}}^{-1} \mathbf{A}_{R}^{\prime} \boldsymbol{j} \boldsymbol{j}^{\prime} \mathbf{A}_{R} \boldsymbol{\Pi}_{\boldsymbol{v}}^{-1}\left(\boldsymbol{\Pi}_{\boldsymbol{v}}^{-1}+\boldsymbol{\Pi}_{\boldsymbol{\epsilon}}^{-1}\right)^{-1}
$$

Using the previous covariance matrix and since $\operatorname{Var}\left[v_{j} \mid p_{R j}, \boldsymbol{s}_{k}\right]=\boldsymbol{j}^{\prime} \boldsymbol{\Pi}_{\boldsymbol{v}}^{-1} \boldsymbol{j}-\left(\operatorname{Cov}\left[v_{j},\left\{p_{R j}, \boldsymbol{s}_{k}\right\}\right]\right)^{\prime}$ $\left(\operatorname{Var}\left[p_{R j}, \boldsymbol{s}_{k}\right]\right)^{-1}\left(\operatorname{Cov}\left[v_{j},\left\{p_{R j}, \boldsymbol{s}_{k}\right\}\right]\right)$, after standard normal calculations I obtain $c_{1 j}=\left(\boldsymbol{j}^{\prime} \mathbf{A}_{R}\left(\boldsymbol{\Pi}_{\boldsymbol{v}}+\right.\right.$ $\left.\left.\boldsymbol{\Pi}_{\boldsymbol{\epsilon}}\right)^{-1} \boldsymbol{j}\right) / D_{1}, \mathbf{c}_{2 j}=\boldsymbol{j}^{\prime}\left(I-\mathbf{A}_{R} c_{1 j}\right)\left(\boldsymbol{\Pi}_{\boldsymbol{v}}+\boldsymbol{\Pi}_{\boldsymbol{\epsilon}}\right)^{-1} \boldsymbol{\Pi}_{\boldsymbol{\epsilon}}$, and, $\operatorname{Var}\left[v_{j} \mid p_{R j}, \boldsymbol{s}_{k}\right]=\boldsymbol{j}^{\prime}\left(I-\mathbf{A}_{R} c_{1 j}\right)\left(\boldsymbol{\Pi}_{\boldsymbol{v}}+\right.$ $\left.\Pi_{\epsilon}\right)^{-1} j$

Proof of proposition 6 Equilibrium existence depends on the existence of a solution to the fixed point problem given by the first of (3.4). To compute the equilibrium, notice that for $j=1,2, i \neq j$, this system can be rewritten as follows:

$$
\begin{aligned}
& a_{R j j}=\gamma \tau_{\epsilon_{j}}-\rho_{\epsilon} a_{R j i} \sqrt{\tau_{\epsilon_{j}} / \tau_{\epsilon_{i}}}, \\
& a_{R j i}=\frac{\gamma}{1-\rho_{\epsilon}^{2}}\left(\tau_{\epsilon_{i}}\left(\frac{h_{j i}}{h_{j j}}\right)-\rho_{\epsilon} \sqrt{\tau_{\epsilon_{j}} \tau_{\epsilon_{i}}}\right),
\end{aligned}
$$

where $a_{R j j}=\left(\mathbf{A}_{R}\right)_{j j}, a_{R j i}=\left(\mathbf{A}_{R}\right)_{j i}, h_{j j}=\left(\left(I-\mathbf{A}_{R} c_{1 j}\right)\left(\boldsymbol{\Pi}_{\boldsymbol{v}}+\boldsymbol{\Pi}_{\boldsymbol{\epsilon}}\right)^{-1}\right)_{j j}$, and $h_{j i}=((I-$ $\left.\left.\mathbf{A}_{R} c_{1 j}\right)\left(\boldsymbol{\Pi}_{\boldsymbol{v}}+\boldsymbol{\Pi}_{\boldsymbol{\epsilon}}\right)^{-1}\right)_{j i}$. To see this, notice that given the above definitions, $\left(\operatorname{Var}\left[v_{j} \mid \boldsymbol{s}_{k}, p_{R j}\right]\right)^{-1}$ $\mathbf{c}_{2 j}=\left(1 \quad\left(h_{j i} / h_{j j}\right)\right) \boldsymbol{\Pi}_{\boldsymbol{\epsilon}}, a_{R j j}=\left(\gamma /\left(1-\rho_{\epsilon}^{2}\right)\right)\left(\tau_{\epsilon_{j}}-\rho_{\epsilon}\left(h_{j i} / h_{j j}\right) \sqrt{\tau_{\epsilon_{j}} \tau_{\epsilon_{i}}}\right)$, and $a_{R j i}=(\gamma /(1-$ 
$\left.\left.\rho_{\epsilon}^{2}\right)\right)\left(\tau_{\epsilon_{i}}\left(h_{j i} / h_{j j}\right)-\rho_{\epsilon} \sqrt{\tau_{\epsilon_{j}} \tau_{\epsilon_{i}}}\right)$. Multiplying both sides of the latter equation by $-\rho_{\epsilon} \sqrt{\tau_{\epsilon_{j}} / \tau_{\epsilon_{i}}}$ and rearranging: $-\rho_{\epsilon} a_{R j i} \sqrt{\tau_{\epsilon_{j}} / \tau_{\epsilon_{i}}}=-\left(\gamma \rho_{\epsilon} /\left(1-\rho_{\epsilon}^{2}\right)\right)\left(\sqrt{\tau_{\epsilon_{j}} \tau_{\epsilon_{i}}}\left(h_{j i} / h_{j j}\right)-\rho_{\epsilon} \tau_{\epsilon_{j}}\right)$. Finally, adding $\gamma \tau_{\epsilon_{j}}$, and simplifying I get (A2).

There are now two cases to consider: the case in which $\rho_{\epsilon}=0$, that gives $a_{R j j}=\gamma \tau_{\epsilon_{j}}$ and a cubic equation in $a_{R j j}$, and the case in which $\rho_{\epsilon} \neq 0$. Start by considering the second (the former is just a simplification of the latter). Substituting the first equation in (A2) into the second one, gives the following cubic equation in $a_{R j i}$

$$
\left(a_{R j i}\right)^{3}\left(1-\rho_{\epsilon}^{2}\right)\left(1-\rho_{v}^{2}\right) \phi_{1}+a_{R j i} \phi_{1} \phi_{2}+\phi_{3}=0
$$

where $\phi_{1}=\tau_{\epsilon_{j}} \tau_{\epsilon_{i}}\left(1-\rho_{v}^{2}\right)+\tau_{v_{j}} \tau_{\epsilon_{i}}+\tau_{v_{i}} \tau_{\epsilon_{j}}+\tau_{v_{j}} \tau_{v_{i}}\left(1-\rho_{\epsilon}^{2}\right)-2 \rho_{\epsilon} \rho_{v} \sqrt{\tau_{\epsilon_{j}} \tau_{\epsilon_{i}} \tau_{v_{j}} \tau_{v_{i}}}, \phi_{2}=\left(\left(1-\rho_{v}^{2}\right) \tau_{\epsilon_{i}}\right.$ $\left.+\left(1-\rho_{\epsilon}^{2}\right) \tau_{v_{i}}+\gamma^{2}\left(1-\rho_{v}^{2}\right) \tau_{\epsilon_{j}} \tau_{\epsilon_{i}} \tau_{u_{j}}\right)$, and $\phi_{3}=\gamma\left(\rho_{\epsilon} \sqrt{\tau_{\epsilon_{j}} \tau_{v_{i}}}-\rho_{v} \sqrt{\tau_{\epsilon_{i}} \tau_{v_{j}}}\right) \sqrt{\tau_{\epsilon_{i}} \tau_{v_{i}}}\left\{\left(\tau_{v_{j}}\left(\tau_{v_{i}}\left(1-\rho_{\epsilon}^{2}\right)+\right.\right.\right.$ $\left.\left.\left.\tau_{\epsilon_{i}}\right)+\tau_{\epsilon_{j}}\left(\tau_{v_{i}}+\tau_{\epsilon_{i}}\left(1-\rho_{v}^{2}\right)\right)\right)-2 \rho_{v} \rho_{\epsilon} \sqrt{\tau_{v_{i}} \tau_{\epsilon_{i}} \tau_{\epsilon_{j}} \tau_{v_{j}}}\right\}$. The discriminant associated to this equation is $\Delta=4\left(\phi_{2} /\left(1-\rho_{\epsilon}^{2}\right)\left(1-\rho_{v}^{2}\right)\right)^{3}+27\left(\phi_{3} /\left(1-\rho_{\epsilon}^{2}\right)\left(1-\rho_{v}^{2}\right) \phi_{1}\right)^{2}$, which can be easily proved to be positive. Hence, there exists a unique real $a_{R j i}$ that satisfies (A3), and the result follows. Q.E.D.

Proof of corollary 2 For part 1, rearranging the cubic equation defining $a_{R j i}$ gives

$$
a_{R j i} \underbrace{\phi_{1}\left(\left(1-\rho_{\epsilon}^{2}\right)\left(1-\rho_{v}^{2}\right)\left(a_{R j i}\right)^{2}+\phi_{2}\right)}_{(1)}+\phi_{3}=0 .
$$

It is easy to check that (1) is positive. Therefore for a solution to exist, it must be the case that $a_{R j i}$ has a sign opposite to $\phi_{3}$. Since $\phi_{3}>0 \Leftrightarrow \tau_{\epsilon_{j}} \operatorname{Cov}\left[\epsilon_{j}, \epsilon_{i}\right]>\tau_{v_{j}} \operatorname{Cov}\left[v_{j}, v_{i}\right]$, the result follows. For part 2, if $\rho_{\epsilon}=0$ the proof is straightforward. Otherwise, assume that $a_{R j j}<0$, then we have $a_{R j i}=\rho_{\epsilon}^{-1} \sqrt{\tau_{\epsilon_{i}} / \tau_{\epsilon_{j}}}\left(\gamma \tau_{\epsilon_{j}}-a_{R j j}\right)$. If $\rho_{\epsilon}>(<) 0, a_{R j i}>(<) 0$ always, a contradiction. Next, 
given the properties of trading intensities, it is easy to see that $\lambda_{R j}=\left(\tau_{u_{j}}\left(a_{R j j}^{2} \tau_{v_{i}}+a_{R j i}^{2} \tau_{v_{j}}+\right.\right.$ $\left.\left.2 \rho_{v} a_{R j j} a_{R j i} \sqrt{\tau_{v_{j}} \tau_{v_{i}}}\right)+\tau_{v_{j}} \tau_{v_{i}}\right)^{-1} \tau_{u_{j}} \tau_{v_{i}}\left(\gamma \tau_{\epsilon_{j}}+a_{R j i}\left(\rho_{v} \sqrt{\tau_{v_{j}} / \tau_{v_{i}}}-\rho_{\epsilon} \sqrt{\tau_{\epsilon_{j}} / \tau_{\epsilon_{i}}}\right)\right)$ is always positive. Finally, parts 3 and 4 follow by manipulating (A2).

Proof of proposition 7 For part 1, I will prove the result by contradiction. Suppose that for $\rho_{v}=0$, $\left|a_{j i}\right| \leq\left|a_{R j i}\right|$. First, let's show that it cannot be that $\left|a_{j i}\right|=\left|a_{R j i}\right|$, for if this was the case then, rewriting (A3)

$$
-\gamma \rho_{\epsilon}\left(\tau_{\epsilon_{j}}\left(\tau_{\epsilon_{i}}+\tau_{v_{i}}\right)+\tau_{v_{j}}\left(\tau_{\epsilon_{i}}+\tau_{v_{i}}\left(1-\rho_{\epsilon}^{2}\right)\right)\right) \frac{\tau_{\epsilon_{j}} \sqrt{\tau_{\epsilon_{i}} \tau_{\epsilon_{j}}}}{\left(1-\rho_{\epsilon}^{2}\right)^{2}}\left(\left(1+\gamma^{2} \tau_{\epsilon_{j}} \tau_{u_{j}}\right)\left(1-\rho_{\epsilon}^{2}\right)+\gamma^{2} \rho_{\epsilon}^{2} \tau_{\epsilon_{j}}\right)
$$

The last equation is null if and only if $\rho_{\epsilon}=0$, hence $\left|a_{j i}\right| \neq\left|a_{R j i}\right|$. Next, suppose $\left|a_{j i}\right|<\left|a_{R j i}\right|$ and choose w.l.o.g. $\rho_{\epsilon}>0$ (i.e. $a_{R j i}<0$ ). Hence, assume $a_{R j i}<-\gamma \rho_{\epsilon} \sqrt{\tau_{\epsilon_{j}} \tau_{\epsilon_{i}}} /\left(1-\rho_{\epsilon}^{2}\right)$. Substituting $-\gamma \rho_{\epsilon} \sqrt{\tau_{\epsilon_{j}} \tau_{\epsilon_{i}}} /\left(1-\rho_{\epsilon}^{2}\right)$ into (A3) its sign should thus be positive. However, as shown by (A4), when $\rho_{\epsilon}>0$, this equation is always negative. A similar argument can be given in the case $\rho_{\epsilon}<0$. Thus, $\left|a_{R j i}\right|>\left|a_{R j i}\right|$. Finally, let us show that $a_{j j}>a_{R j j}$. Consider again $\rho_{\epsilon}>0$. I have just shown that in this case either $a_{R j i}>-\gamma \rho_{\epsilon} \sqrt{\tau_{\epsilon_{j}} \tau_{\epsilon_{i}}} /\left(1-\rho_{\epsilon}^{2}\right)$ or $-a_{R j i}<\gamma \rho_{\epsilon} \sqrt{\tau_{\epsilon_{j}} \tau_{\epsilon_{i}}} /\left(1-\rho_{\epsilon}{ }^{2}\right)$. Multiply both sides of the last inequality by $\rho_{\epsilon} \sqrt{\tau_{\epsilon_{j}} / \tau_{\epsilon_{i}}}$ and add $\gamma \tau_{\epsilon_{j}}$. Rearranging this gives $a_{R j j} \equiv \gamma \tau_{\epsilon_{j}}-\rho_{\epsilon} a_{R j i} \sqrt{\tau_{\epsilon_{j}} / \tau_{\epsilon_{i}}}<\gamma \tau_{\epsilon_{j}} /\left(1-\rho_{\epsilon}{ }^{2}\right)$. A similar argument can be given for $\rho_{\epsilon}<0$. Hence, $a_{j j}>a_{R j j}$ and the result follows. For part 2, if $\rho_{\epsilon}=0$ then $a_{j i}=0$, while $\left|a_{R j i}\right|>0$, hence $\left|a_{R j i}\right|>\left|a_{j i}\right|$, whereas $a_{R j j}=a_{j j}=\gamma \tau_{\epsilon_{j}}$. For part 3, if $\rho_{\epsilon}=\rho_{v}=0$, trading intensities coincide across the two systems.

Proof of proposition 8 Suppose $\rho_{\epsilon}=\rho_{u}=0$, and set $a_{1}=\mathbf{A}_{11}=\mathbf{A}_{22}, a_{2}=\mathbf{A}_{12}=\mathbf{A}_{21}, a_{R 1}=$ $\left(\mathbf{A}_{R}\right)_{11}=\left(\mathbf{A}_{R}\right)_{22}$, and $a_{R 2}=\left(\mathbf{A}_{R}\right)_{12}=\left(\mathbf{A}_{R}\right)_{21}$. Straightforward normal calculations in the homoscedastic case give $I_{p}=\tau_{v}^{-1}-\left(\tau_{v}^{2}+a_{1}^{4} \tau_{u}^{2}\left(1-\rho_{v}^{2}\right)+2 a_{1}^{2} \tau_{u} \tau_{v}\right)^{-1}\left(\tau_{v}+a_{1}^{2} \tau_{u}\left(1-\rho_{v}^{2}\right)\right)$, and $I_{p_{R}}=$ 
$\tau_{v}^{-1}-\left(\tau_{v}^{2}+\tau_{u}^{2}\left(1-\rho_{v}^{2}\right)\left(a_{R 1}^{2}-a_{R 2}^{2}\right)^{2}+2 \tau_{u} \tau_{v}\left(a_{R 1}^{2}+a_{R 2}^{2}+2 \rho_{v} a_{R 1} a_{R 2}\right)\right)^{-1}\left(\tau_{v}+\left(a_{R 1}^{2}+a_{R 2}^{2}\right) \tau_{u}(1-\right.$ $\left.\rho_{v}^{2}\right)$ ). Implicitly differentiating (A3) with respect to $\rho_{v}$, one can see that $I_{p_{R}}$ is convex in $\rho_{v}$ and has a local minimum in $\rho_{v}=0$. The same result can be obtained for $I_{p}$. Perform a second order expansion of $I_{p_{R}}$ and $I_{p}$ around $\rho_{v}=0$ to get $I_{p_{R}}\left(\rho_{v}\right)=I_{p_{R}}(0)+\left.\left(\rho_{v}^{2} / 2\right)\left(\partial^{2} I_{p_{R}} / \partial \rho_{v}^{2}\right)\right|_{\rho_{v}=0}+R_{1}(0)$ and $I_{p}=I_{p}(0)+\left.\left(\rho_{v}^{2} / 2\right)\left(\partial^{2} I_{p} / \partial \rho_{v}^{2}\right)\right|_{\rho_{v}=0}+R_{2}(0)$, where $\left.\left(\partial^{2} I_{p_{R}} / \partial \rho_{v}^{2}\right)\right|_{\rho_{v}=0}=\left(2 a_{R 1}^{2} \tau_{u} \tau_{v}\left(\left(\tau_{\epsilon}+\right.\right.\right.$ $\left.\left.\left.a_{R 1}^{2} \tau_{u}\right)^{2}+3 \tau_{v}\left(\tau+2 \tau_{\epsilon}+\tau_{v}\right)\right)\right) /\left(\tau^{3}\left(\tau+\tau_{\epsilon}\right)^{2}\right),\left.\left(\partial^{2} I_{p} / \partial \rho_{v}^{2}\right)\right|_{\rho_{v}=0}=\left(2 a_{1}^{2} \tau_{u} \tau_{v}\right) / \tau^{3}$ and $\tau=\tau_{v}+a_{1}^{2} \tau_{u}$,

$a_{1}=\gamma \tau_{\epsilon}$. As for $\rho_{v}=0, \mathbf{A}=\mathbf{A}_{R}$ then $I_{p_{R}}(0)=I_{p}(0)$, and $I_{p_{R}}-I_{p}=\left(\rho_{v}^{2} / 2\right)\left(\tau^{3}(\tau+\right.$ $\left.\left.\tau_{\epsilon}\right)^{2}\right)^{-1}\left(2 a_{1}^{2} \tau_{u} \tau_{v}^{2}\left(4 \tau_{\epsilon}+a_{1}^{2} \tau_{u}+5 \tau_{v}\right)\right)$, which is always positive. The result follows.

Q.E.D.

Proof of proposition 9 Using the same notation of the previous proof, suppose $\rho_{\epsilon}=\rho_{u}=0$. Perform a second order Taylor expansion of $\lambda_{R}\left(\rho_{v}\right)=\left(\tau_{u}\left(a_{R 1}^{2}+a_{R 2}^{2}+2 \rho_{v} a_{R 1} a_{R 2}\right)+\tau_{v}\right)^{-1}$ $\left(\tau_{u}\left(\gamma_{\epsilon}+a_{R 2} \rho_{v}\right)\right)$ around $\rho_{v}=0$, then $\lambda_{R}\left(\rho_{v}\right)=\lambda_{R}(0)+\left.\left(\rho_{v}^{2} / 2\right)\left(\partial^{2} \lambda_{R} / \partial \rho_{v}^{2}\right)\right|_{\rho_{v}=0}+R_{1}(0)$, where $\left.\left(\partial^{2} \lambda_{R} / \partial \rho_{v}^{2}\right)\right|_{\rho_{v}=0}=-\left(\tau^{2}\left(\tau+\tau_{\epsilon}\right)^{2}\right)^{-1}\left(2 \gamma \tau_{\epsilon} \tau_{u} \tau_{v}\left(a_{1}^{4} \tau_{u}^{2}+a_{1}^{2} \tau_{u}\left(\tau_{\epsilon}+\tau_{v}\right)-\tau_{v}\left(\tau_{\epsilon}+\tau_{v}\right)\right)\right.$. In the same way, for the unrestricted system $\lambda\left(\rho_{v}\right)=\lambda(0)+\left.\left(\rho_{v}^{2} / 2\right)\left(\partial^{2} \lambda / \partial \rho_{v}^{2}\right)\right|_{\rho_{v}=0}+R_{2}(0)$, where $\left.\left(\partial^{2} \lambda / \partial \rho_{v}^{2}\right)\right|_{\rho_{v}=0}=-\tau^{-3} 2 a_{1}^{3} \tau_{u}^{2} \tau_{v}$. As $\lambda_{R}(0)=\lambda(0)$, and $\lambda_{R}\left(\rho_{v}\right)-\lambda\left(\rho_{v}\right)=\left(\rho_{v} / 2\right)^{2}\left(\tau^{3}(\tau+\right.$ $\left.\left.\tau_{\epsilon}\right)^{2}\right)^{-1}\left(2 \gamma \tau_{\epsilon} \tau_{u} \tau_{v}\left(a_{1}^{4} \tau_{u}^{2} \tau_{\epsilon}+\tau_{v}^{2}\left(\tau_{\epsilon}+\tau_{v}\right)+a_{1}^{2} \tau_{u}\left(\tau_{\epsilon}+\tau_{v}\right)^{2}\right)\right)>0$, the result follows. Q.E.D.

\section{Appendix B}

First of all, I state a well known result on multivariate normal random variables (see e.g. Danthine and Moresi, 1992).

Lemma Bl Let $\boldsymbol{w}$ be a vector of $n$ random variables. Assume $\boldsymbol{w} \sim N(\boldsymbol{\mu}, \boldsymbol{\Sigma})$, with $\boldsymbol{\Sigma}$ non singular. Define $Q(\boldsymbol{w})=D+\boldsymbol{b}^{\prime} \boldsymbol{w}+\boldsymbol{w}^{\prime} \mathbf{F} \boldsymbol{w}$, where $D \in \Re, \boldsymbol{b} \in \Re^{n}$, and $\mathbf{F}$ is a symmetric $(n \times n)$ matrix. 
Then if $2 \mathbf{F}+\boldsymbol{\Sigma}^{-1}$ is positive definite

$$
\begin{aligned}
E[\exp (Q(\boldsymbol{w}))]=|\boldsymbol{\Sigma}|^{-1 / 2}\left|2 \mathbf{F}+\boldsymbol{\Sigma}^{-1}\right|^{-1 / 2} \times \\
\quad \exp \left\{D+\boldsymbol{b}^{\prime} \boldsymbol{\mu}+\boldsymbol{\mu}^{\prime} \mathbf{F} \boldsymbol{\mu}+\frac{1}{2}(\boldsymbol{b}-\mathbf{F} \boldsymbol{\mu})^{\prime}\left(2 \mathbf{F}+\boldsymbol{\Sigma}^{-1}\right)^{-1}(\boldsymbol{b}-\mathbf{F} \boldsymbol{\mu})\right\} .
\end{aligned}
$$

Determination of the informed ex-ante expected utility in the restricted system.

Notice that a trader $k$ 's strategy in both assets, can be expressed as $\mathbf{X}_{R k}\left(\boldsymbol{s}_{k}, \boldsymbol{p}_{R}\right)=\mathbf{A}_{R}\left(\boldsymbol{s}_{k}-\right.$ $\overline{\boldsymbol{v}})+\mathbf{B}\left(\boldsymbol{v}-\boldsymbol{p}_{R}\right)$, where

$$
\mathbf{A}_{R}=\left(\begin{array}{cc}
a_{R 11} & a_{R 12} \\
a_{R 21} & a_{R 22}
\end{array}\right), \quad \mathbf{B}=\left(\begin{array}{cc}
b_{R 1} & 0 \\
0 & b_{R 2}
\end{array}\right)
$$

Next, standard normal computations give

$$
E\left[\pi_{R k} \mid \boldsymbol{v}, \boldsymbol{p}_{R}\right]-(1 / 2 \gamma) \operatorname{Var}\left[\pi_{R k} \mid \boldsymbol{v}, \boldsymbol{p}_{R}\right]=\left(\begin{array}{c}
\boldsymbol{v}-\boldsymbol{p}_{R} \\
\overline{\boldsymbol{v}}-\boldsymbol{p}_{R}
\end{array}\right)^{\prime} \boldsymbol{\Omega}\left(\begin{array}{c}
\boldsymbol{v}-\boldsymbol{p}_{R} \\
\overline{\boldsymbol{v}}-\boldsymbol{p}_{R}
\end{array}\right)
$$

where $\pi_{R k}=\boldsymbol{x}_{R k}^{\prime}\left(\boldsymbol{v}-\boldsymbol{p}_{R}\right)$,

$$
\boldsymbol{\Omega}=\left(\begin{array}{cc}
\mathbf{A}_{R}^{\prime}-(1 / 2 \gamma) \mathbf{A}_{R} \boldsymbol{\Pi}_{\boldsymbol{\epsilon}}^{-1} \mathbf{A}_{R}^{\prime} & (1 / 2)\left(\mathbf{B}^{\prime}-\mathbf{A}_{R}^{\prime}\right) \\
(1 / 2)\left(\mathbf{B}^{\prime}-\mathbf{A}_{R}^{\prime}\right) & \mathbf{0}
\end{array}\right), \quad\left(\begin{array}{c}
\boldsymbol{v}-\boldsymbol{p}_{R} \\
\overline{\boldsymbol{v}}-\boldsymbol{p}_{R}
\end{array}\right) \sim N(\mathbf{0}, \mathbf{\Psi}),
$$


and

$\Psi=$

$\left(\begin{array}{cc}\left(I-\boldsymbol{\Lambda}_{R} \mathbf{A}_{R}\right) \boldsymbol{\Pi}_{\boldsymbol{v}}^{-1}\left(I-\boldsymbol{\Lambda}_{R} \mathbf{A}_{R}\right)^{\prime}+\boldsymbol{\Lambda}_{R} \boldsymbol{\Pi}_{\boldsymbol{u}}^{-1} \boldsymbol{\Lambda}_{R}^{\prime} & -\left(\left(I-\boldsymbol{\Lambda}_{R} \mathbf{A}_{R}\right) \boldsymbol{\Pi}_{\boldsymbol{v}}^{-1} \mathbf{A}_{R}^{\prime}+\boldsymbol{\Lambda}_{R} \boldsymbol{\Pi}_{\boldsymbol{u}}^{-1}\right) \boldsymbol{\Lambda}_{R}^{\prime} \\ -\boldsymbol{\Lambda}_{R}\left(\left(I-\boldsymbol{\Lambda}_{R} \mathbf{A}_{R}\right) \boldsymbol{\Pi}_{\boldsymbol{v}}^{-1} \mathbf{A}_{R}^{\prime}+\boldsymbol{\Lambda}_{R} \boldsymbol{\Pi}_{\boldsymbol{u}}^{-1}\right)^{\prime} & \boldsymbol{\Lambda}_{R}\left(\mathbf{A}_{R} \boldsymbol{\Pi}_{\boldsymbol{v}}^{-1} \mathbf{A}_{R}^{\prime}+\boldsymbol{\Pi}_{\boldsymbol{u}}^{-1}\right) \boldsymbol{\Lambda}_{R}^{\prime}\end{array}\right)$

Notice that $E\left[-\exp \left\{-\gamma^{-1} \pi_{R k}\right\} \mid \boldsymbol{v}, \boldsymbol{p}_{R}\right]=-\exp \left\{-\gamma^{-1}\left(E\left[\pi_{R k} \mid \boldsymbol{v}, \boldsymbol{p}_{R}\right]-(1 / 2 \gamma) \operatorname{Var}\left[\pi_{R k} \mid \boldsymbol{v}, \boldsymbol{p}_{R}\right]\right)\right\}$, and $E\left[-\exp \left\{-\gamma^{-1} \pi_{R k}\right\}\right]=E\left[E\left[-\exp \left\{-\gamma^{-1} \pi_{R k}\right\} \mid \boldsymbol{v}, \boldsymbol{p}_{R}\right]\right]$. As $\boldsymbol{\Psi}$ can be checked to be non singular, applying lemma B1 with $\left(\left(\boldsymbol{v}-\boldsymbol{p}_{R}\right)^{\prime},\left(\overline{\boldsymbol{v}}-\boldsymbol{p}_{R}\right)^{\prime}\right)^{\prime}=\boldsymbol{w}$, one finds $E\left[-\exp \left\{-\gamma^{-1} \pi_{R k}\right\}\right]$ $=-|\boldsymbol{\Psi}|^{-1 / 2}\left|(2 / \gamma) \boldsymbol{\Omega}+\boldsymbol{\Psi}^{-1}\right|^{-1 / 2}$. 


\section{References}

Admati, A.R. "A Noisy Rational Expectations Equilibrium for Multi-Asset Securities Markets.” Econometrica, Vol. 53 (1985), pp. 629-657.

Aminud, Y. And Mendelson, H. "Trading Mechanisms and Value Discovery: CrossNational Evidence and Implications." Carnegie-Rochester Conference Series on Public Policy, Vol. 0 (1991a), pp. 105-130.

— AND — . "How (Not) to Integrate the European Capital Markets." In Giovannini and Mayer, eds., European Financial Integration, Cambridge: Cambridge University Press, $1991 b$.

Beja, A. And Hakansson, N.H. "From Orders to Trades: Some Alternative Market Mechanisms.” In E. Bloch and R. Schwartz, eds., Impending Changes for Securities Markets: what Role for the Exchanges?, Greenwich: JAI Press, 1979.

BIAIS, B. "Price Formation and Equilibrium Liquidity in Fragmented and Centralized Markets." Journal of Finance, Vol. 48 (1993), pp. 105-124.

Bossaerts, P., Fine, L., And Ledyard J. "Inducing Liquidity in Thin Financial Markets Through Combined-Value Mechanisms.” European Economic Review, Vol. 46 (2002), pp. 1671-1695.

Brown, D.P. AND Holden, C.W. “Adjustable Limit Orders.” Working Paper, Kelley School of Business, Indiana University, 2002.

CABAllé, J. ANd Krishnan, M. "Imperfect Competition in a Multi-Security Market with Risk Neutrality.” Econometrica, Vol. 62 (1994), pp. 695-704.

Cespa, G. "Short-term Investment and Equilibrium Multiplicity." European Economic Review, Vol. 46 (2002), pp. 1645-1670.

Cespa, G. "Giffen Goods and Market Making." Working Paper no. 681, Departament d'Economia i Empresa, Universitat Pompeu Fabra, 2003.

CHAN, K. "Imperfect Information and Cross-Autocorrelation Among Stock Prices." Journal of Finance, Vol. 48 (1992), pp. 1211-1230.

Clemons, E.K. AND Weber, B.W. "Restructuring Institutional Block Trading: an Overview of the Optimark System.” Journal of Management Information Systems, Vol. 15 (1998), pp. 41-60.

Danthine, J.-P. AND Moresi, S. "Volatility, Information, and Noise Trading." European Economic Review, Vol. 37 (1992), pp. 961-982.

DeGroot, M.H. Optimal Statistical Decisions. New York: McGraw-Hill, 1969.

Diamond, D. And Verrecchia, R.E. "Information Aggregation in a Noisy Rational Expectations Economy." Journal of Financial Economics, Vol. 9 (1981), pp. 221-235.

Dow, J. AND RAHI, R. "Informed Trading, Investment, and Welfare." Journal of Business (Forthcoming), (2002).

EConomides, N. And Schwartz, R.A. "Electronic Call Market Trading." Journal of Portfolio Management, Vol. 21 (1995), pp. 10-18. 
Fishman, M.J. And Hagerty, K.M. "Disclosure Decisions by Firms and the competition for price efficiency." Journal of Finance, Vol. 44 (1989), pp. 633-646.

— AND — . "Insider Trading and the Efficiency of Stock Prices." RAND Journal of Economics, Vol. 23 (1992), pp. 106-122.

Foucault, T. "Order Flow Composition and Trading Costs is a Dynamic Limit Order Market." Journal of Financial Markets, Vol. 2 (1999), pp. 99-134.

Gertner, R., Gibbons, R., And Scharfstein, D. "Simultaneous Signalling to the Capital and Product Market." RAND Journal of Economics, Vol. 19 (1988), pp. 173-190.

Grossman, S.J. “The Informational Role of Upstairs and Downstairs Trading.” Journal of Business, Vol. 65 (1992), pp. 509-528.

— AND Stiglitz, J. "On the Impossibility of Informationally Efficient Markets.” American Economic Review, Vol. 70 (1980), pp. 393-408.

HE, H. AND WAng, J. "Differential Information and Dynamic Behavior of Stock Trading Volume.” Review of Financial Studies, Vol. 8 (1995), pp. 919-972.

HeLlwig, M.F. "On the Aggregation of Information in Competitive Markets." Journal of Economic Theory, Vol. 22 (1980), pp. 477-498.

Kyle, A. "Continuous Auctions and Insider Trading." Econometrica, Vol. 53 (1985), pp. 1315-1336.

Lindsay, R. And Schaede, U. "Specialist vs. Saitori: Market Making in New York and Tokyo." Haas School of Business Finance Working Paper 202, (1990).

Madhavan, A. "Trading Mechanisms in Securities Markets." Journal of Finance, Vol. 47 (1992), pp. 607-642.

ManZANo, C. "Informed Speculation with Imperfect Competition in a Multi-Security Market." Ph.D. thesis, Universitat Autònoma de Barcelona (1997).

Pagano, M. AND RöEll, A. "Transparency and Liquidity: a Comparison of Auction and Dealer Markets with Informed Trading." Journal of Finance, Vol. 51 (1996), pp. 580-611.

Palomino, F. "Informational Efficiency: Ranking Markets." Economic Theory, Vol. 18 (2001), pp. 683-700.

Poitevin, M. "Financial Signalling and the "Deep-Pocket" Argument." RAND Journal of Economics, Vol. 20 (1989), pp. 26-40.

Rochet, J.C. AND VILA, J.L. "Insider Trading Without Normality." Review of Economic Studies, Vol. 61 (1994), pp. 131-152.

Tong, Y. The Multivariate Normal Distribution. New York: Springer-Verlag, 1990.

VIVES, X. "The Speed of Information Revelation in a Financial Market.” Journal of Economic Theory, Vol. 67 (1995a), pp. 178-204.

—. "Short-Term Investment and the Informational Efficiency of the Market." Review of Financial Studies, Vol. 8 (1995b), pp. 125-160.

Wohl, A. AND Kandel, S. "Implications of an Index-Contingent Trading Mechanism." Journal of Business, Vol. 70 (1997), pp. 471-488. 


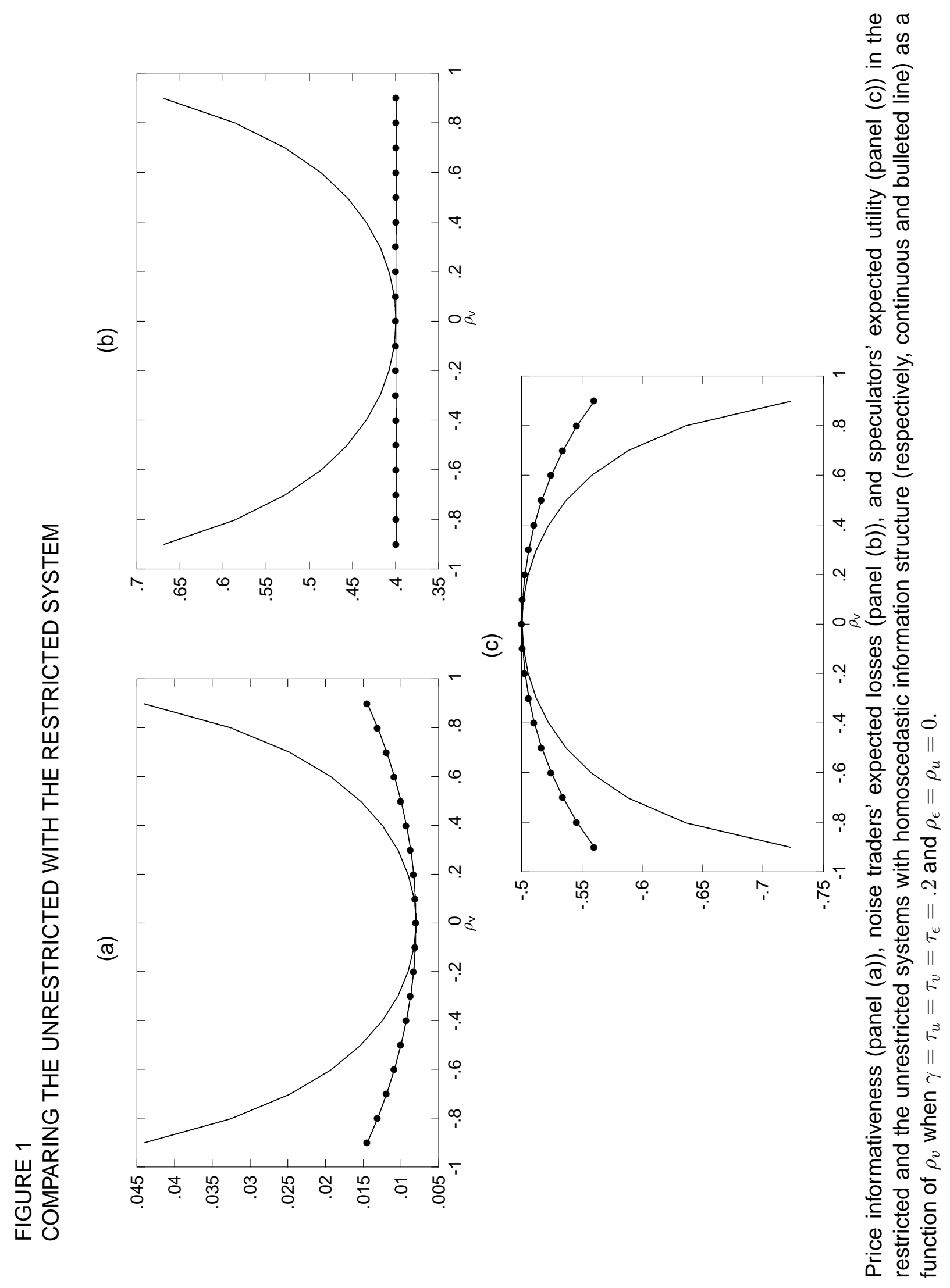




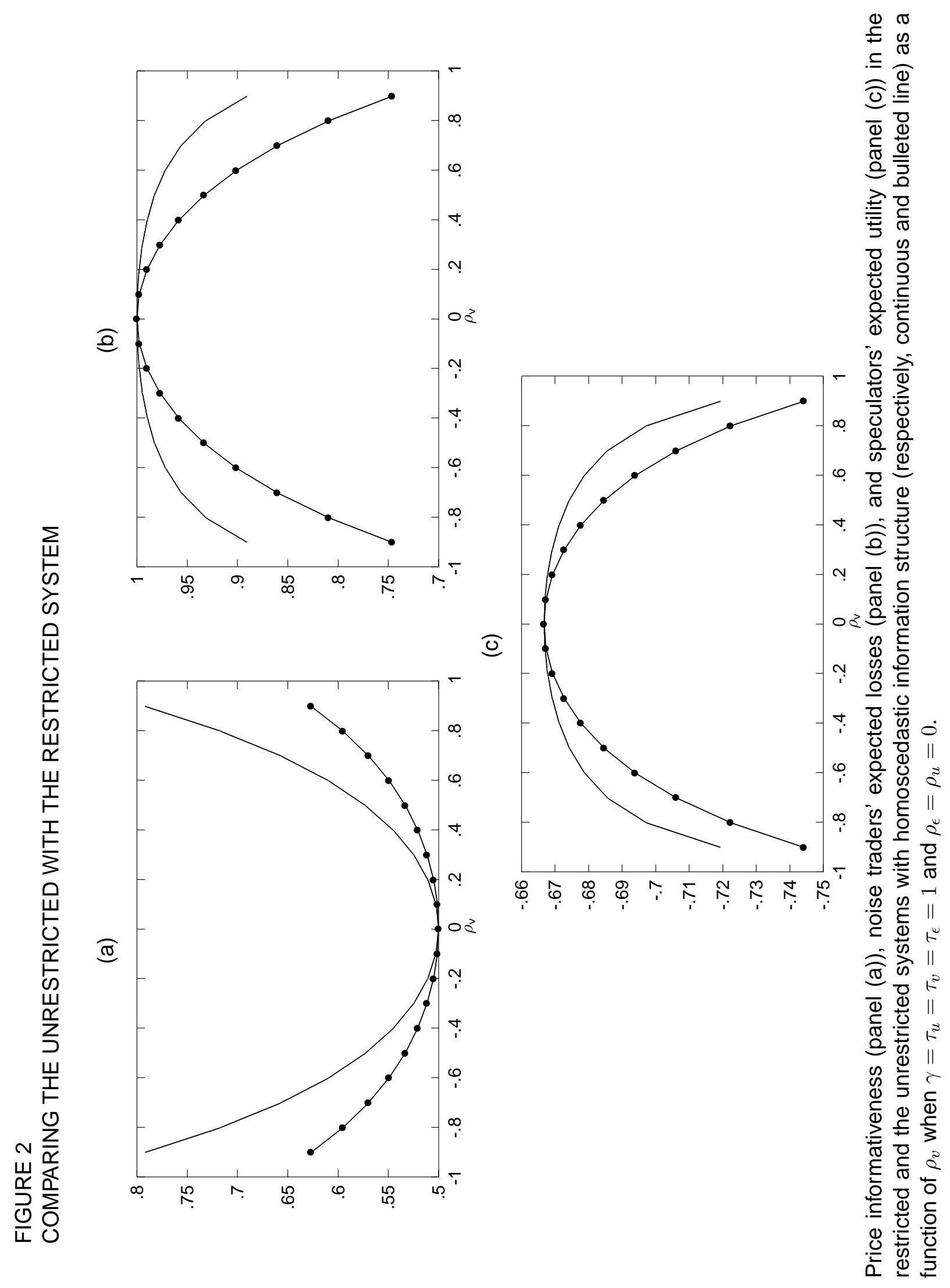




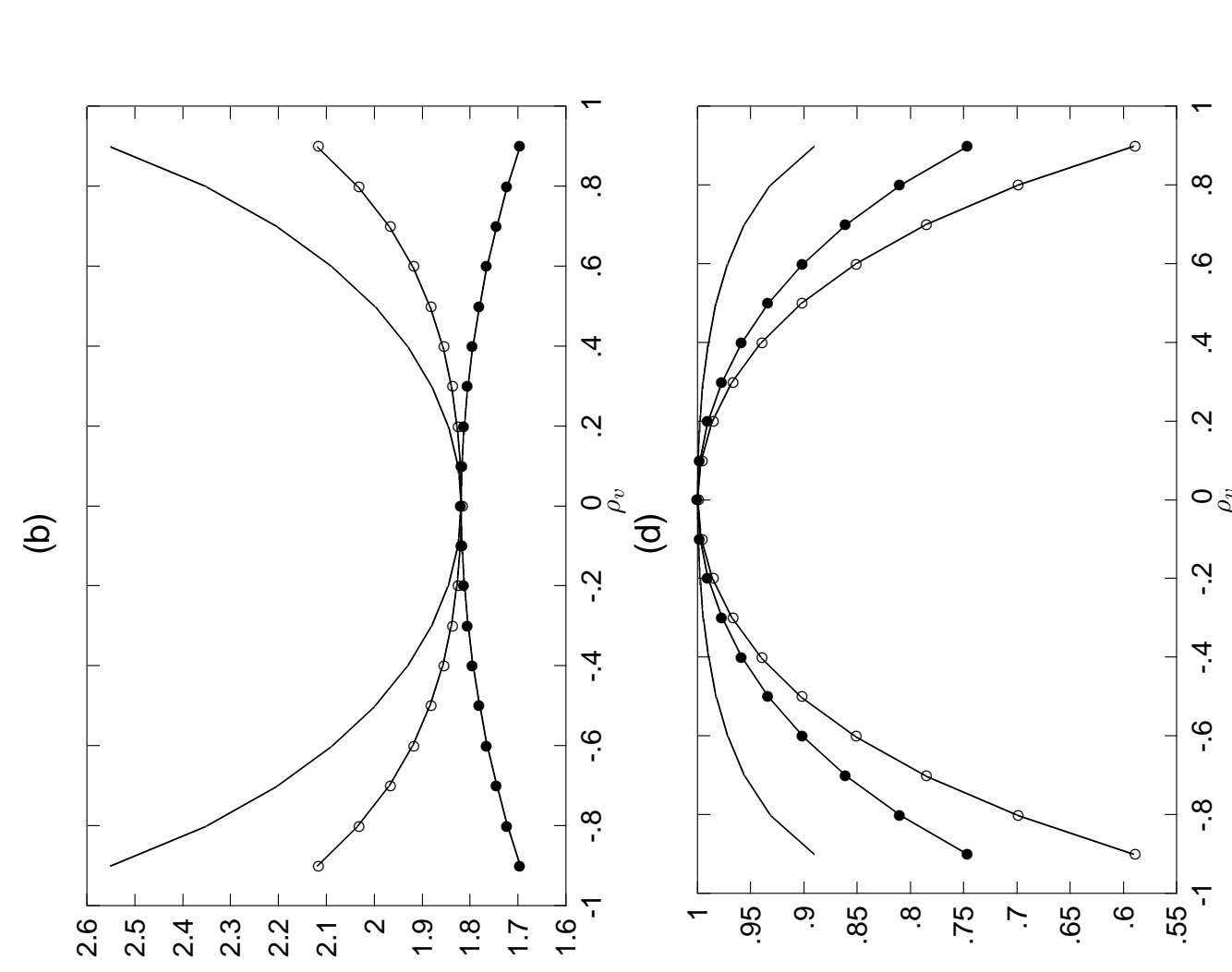

오 응

닥 3 है $\left.\frac{\Phi}{0}\right)^{2}$ के 1

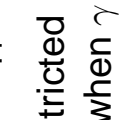
के 는 등 을 甲

娄 造 ब .

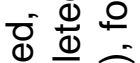
은

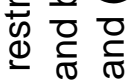
$\stackrel{0}{\circ}$ (0)

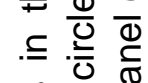
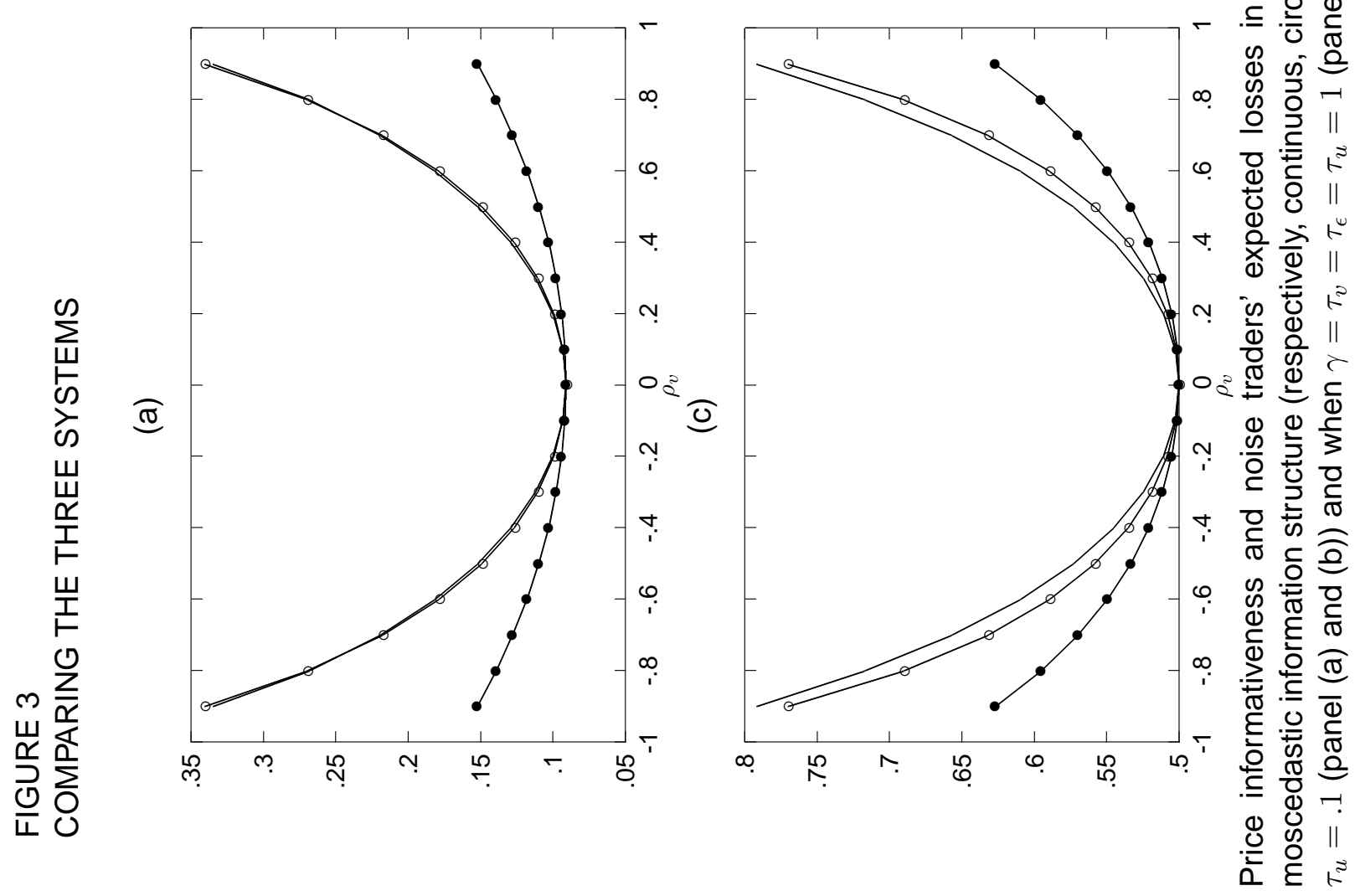